Check for updates

Cite this: RSC Adv., 2018, 8, 21495

Received 30th April 2018 Accepted 3rd June 2018

DOI: $10.1039 / c 8 r a 03703 e$

rsc.li/rsc-advances

\title{
A one-pot, multicomponent reaction for the synthesis of novel 2-alkyl substituted 4- aminoimidazo[1,2-a][1,3,5]triazines $\dagger+$
}

\author{
Felicia Phei Lin Lim, (D) ${ }^{a}$ Lin Yuing Tan, (D) ${ }^{a}$ Edward R. T. Tiekink ${ }^{b}$ \\ and Anton V. Dolzhenko (iD *ac
}

\begin{abstract}
A highly selective, one-pot, three-component synthesis of novel 2-alkyl-substituted 4-aminoimidazo[1,2-a] $[1,3,5]$ triazines has been developed. The scope of the method was explored in two dimensions, varying the structures of trialkyl orthoesters and 2-aminoimidazoles in their reactions with cyanamide. Conveniently performed under microwave irradiation, this method was also proved to be efficient under conventional heating. A library of 24 novel compounds was prepared in high purity using this multicomponent approach. Molecular and crystal structures of representative molecules were studied using X-ray crystallography.
\end{abstract}

\section{Introduction}

The imidazo[1,2-a][1,3,5]triazine ring system is recognised as an important scaffold in medicinal chemistry. ${ }^{2}$ The well-defined targets for imidazo[1,2-a][1,3,5]triazines include a variety of enzymes, such as focal adhesion kinase, ${ }^{3}$ activated Cdc42associated tyrosine kinase $1,{ }^{4}$ dipeptidyl peptidase IV, ${ }^{5}$ and phosphodiesterase $5 .^{6}$ This scaffold has been successfully used for the design of ligands for $\mu$-opioid ${ }^{7}$ and $\mathrm{A}_{1}$ adenosine $^{8}$ receptors. The pharmacological potential of imidazo[1,2-a] $[1,3,5]$ triazines as anti-cancer, ${ }^{9}$ anti-viral ${ }^{10}$ and anti-diabetic ${ }^{11}$ agents has also been well documented in the literature. The broad spectrum of biological activities of imidazo[1,2-a][1,3,5] triazines results in ongoing demand for the development of new practical methods for the synthesis of diversely functionalized imidazo[1,2- $a][1,3,5]$ triazines.

Since the first report ${ }^{12}$ on the synthesis of the imidazo[1,2-a] $[1,3,5]$ triazine ring system 50 years ago, a number of methods for the preparation of compounds with this heterocyclic core have been reported. They typically involve triazine ring

\footnotetext{
${ }^{a}$ School of Pharmacy, Monash University Malaysia, Jalan Lagoon Selatan, Bandar Sunway, Selangor Darul Ehsan 47500, Malaysia. E-mail: dolzhenkoav@gmail.com; anton.dolzhenko@monash.edu; Fax: +60-3-5514-6364; Tel: +60-3-5514-5867

${ }^{b}$ Research Centre for Crystalline Materials, School of Science and Technology, Sunway University, Bandar Sunway, Selangor Darul Ehsan 47500, Malaysia

${ }^{c}$ School of Pharmacy and Biomedical Sciences, Curtin Health Innovation Research Institute, Faculty of Health Sciences, Curtin University, GPO Box U1987 Perth, Western Australia 6845, Australia

$\dagger$ Part 33 in the series "Fused heterocyclic systems with an $s$-triazine ring." For part 32 see ref. 1 .

\$ Electronic supplementary information (ESI) available. CCDC 1840247 and 1840248. For ESI and crystallographic data in CIF or other electronic format see DOI: $10.1039 / \mathrm{c} 8 \mathrm{ra03703e}$
}

construction onto substituted imidazoles ${ }^{13}$ or, more often, the annulation of the imidazole ring onto substituted 1,3,5triazines. ${ }^{14}$ The two most common approaches utilize: (1) reactions of 2-amino-1,3,5-triazines with $\alpha$-haloaldehydes/ketone$\mathrm{s}^{3,8,12,14 a, b}$ or chalcones, ${ }^{14 c}$ and (2) intramolecular cyclisations of

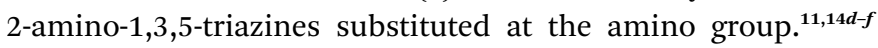
Reports on the preparation of imidazo[1,2-a][1,3,5]triazines via the formation of the 1,3,5-triazine ring onto imidazoles are limited and usually utilize addition of 2-aminoimidazoles to $\mathrm{N}$ acyliso(thio)cyanates followed by the intramolecular closure of the $1,3,5$-triazine ring. ${ }^{13}$

Multicomponent reactions (MCRs) represent an efficient strategy in modern synthetic chemistry. ${ }^{15}$ Minimising the number of synthetic steps in obtaining targeted compounds from available starting reagents is highly desirable in the fields of organic synthesis and drug discovery. Due to their diversity and combinatorial potential, MCRs have become highly appreciated tools for the preparation of libraries of compounds for biological screening. ${ }^{16}$ However, issues surrounding chemoand regio-selectivity of MCRs often pose a challenge and require fine-tuning during method development. The need for rapid and selective construction of biologically active compounds for drug discovery has stimulated intense development in the use of microwave technology. Microwave irradiation has been effectively used as an alternative source of energy, improving efficiency and selectivity of MCRs. ${ }^{1,17,18}$

We have been exploring the potential for new one-pot, multicomponent, microwave-assisted protocols for the synthesis of azolo[ $[1,3,5]$ triazines..$^{18}$ After the development of the MCRs utilizing aminoazoles, orthoformates and cyanamide, we have been refining the scope of this reaction using a variety of aminoazoles. In particular, the molecular diversity of compounds prepared has been achieved by varying the 
structures of the aminoazole precursors used in this threecomponent condensation and included libraries of pyrazolo $[1,5-a][1,3,5]$ triazines, ${ }^{1,18 a-c} \quad$ 1,2,4-triazolo[1,5- $\left.a\right][1,3,5]$ triazines, ${ }^{18 d, e}$ and imidazo[1,2-a][1,3,5]triazines. ${ }^{18 f}$ Herein, we report our attempts to further expand the scope of this MCR by exploring its capacity to accommodate various orthoesters and therefore, introduce a new point of diversity in the reaction products. 2-Aminoimidazoles were selected as aminoazole substrates for the reaction in order to explore the regio- and chemo-selectivity of the process for these challenging substrates bearing endocyclic nitrogen atoms of similar reactivity.

\section{Results \& discussion}

\section{Synthesis}

The starting materials used in our three-component reaction, viz. 2-aminoimidazoles (1), were prepared using the previously reported method developed by Van der Eycken's group. ${ }^{19}$ Optimization of the conditions for our proposed MCR was performed using a model reaction of 2-amino-4-phenylimidazole (1a), triethyl orthoacetate, and cyanamide under microwave irradiation (Table 1). The initial attempt to carry out this reaction at $150{ }^{\circ} \mathrm{C}$ for $20 \mathrm{~min}$ in ethyl acetate led to 4-amino-2methyl-7-phenylimidazo[1,2-a][1,3,5]triazine (5a) obtained in $42 \%$ yield. Despite the moderate yield, the product 5a was isolated in a chromatography-free process as the sole product. We found that the yield could be improved to $60 \%$ by performing the reaction at $160{ }^{\circ} \mathrm{C}$ for $35 \mathrm{~min}$. However, despite attempts to further increase the yields by manipulating the reaction conditions, no improvement in the outcome was achieved.

The developed method was tested using two different models of microwave synthesizers: Discover SP (CEM) and Monowave 400 (Anton Paar). The three-component reaction of 1a, triethyl orthoacetate and cyanamide under the optimized

Table 1 Optimization of conditions for the model reaction ${ }^{a}$

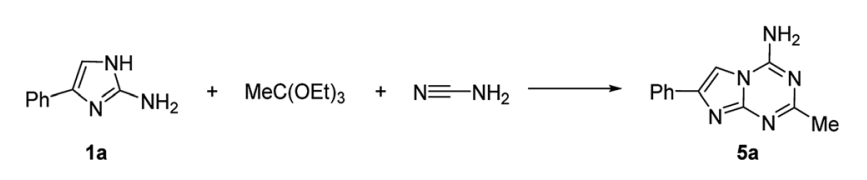

\begin{tabular}{llll}
\hline Entry & Temperature $\left({ }^{\circ} \mathrm{C}\right)$ & Time $(\mathrm{min})$ & Isolated yield $(\%)$ \\
\hline 1 & 150 & 20 & 42 \\
2 & 150 & 30 & 47 \\
3 & 160 & 20 & 47 \\
4 & 170 & 20 & 41 \\
5 & 160 & 30 & 51 \\
6 & 160 & 35 & 60 \\
7 & 160 & 40 & 47 \\
$8^{b}$ & 160 & 35 & 45
\end{tabular}

${ }^{a}$ The reaction was performed using a Discover SP CEM microwave synthesizer with $1 \mathrm{mmol}$ of $1 \mathrm{a}, 2.5 \mathrm{mmol}$ of triethyl orthoacetate and $2.5 \mathrm{mmol}$ of cyanamide in $2 \mathrm{~mL}$ of the ethyl acetate. ${ }^{b}$ The reaction was performed using $1 \mathrm{mmol}$ of $1 \mathrm{a}, 3 \mathrm{mmol}$ of triethyl orthoacetate and $3 \mathrm{mmol}$ of cyanamide. conditions was conducted in both systems and resulted in the formation of $5 \mathrm{a}$ in $60 \%$ and $56 \%$ yields, respectively.

Attempts to perform the reaction under reflux in ethyl acetate for $12 \mathrm{~h}$ led to the recovery of the starting material 1a without traces of 5a. However, an attempt to carry out the MCR of 1a, triethyl orthoacetate and cyanamide in the Monowave 50 (Anton Paar) reactor using sealed vessels under fast conventional heating imitating the optimised microwave irradiation conditions was successful, resulting in the 54\% yield of equally pure 5a. Therefore, we could exclude any significant contribution of non-thermal microwave effects in promoting this MCR.

In principle, the annulation of the 1,3,5-triazine ring onto the 2-aminoimidazole $1 \mathrm{a}$ in the three-component reaction could proceed with the formation of four regioisomeric structures viz. 2-aminoimidazo[1,2- $a][1,3,5]$ triazines 2 and 3 as well as 4 -aminoimidazo[1,2- $a][1,3,5]$ triazines $\mathbf{4}$ and $\mathbf{5 a}$ (Scheme 1). However, only one product was isolated from the MCR. The structure of this product was assigned on the basis of spectral data and an alternative synthesis of $\mathbf{5 a}$ adopting our previously reported step-wise synthesis of azolo[1,3,5]triazines via the 1,3,5-triazine ring annulation onto aminoazoles with a predisposed position of the amino group on the triazine ring. ${ }^{20}$ This method converted aminoazoles into formamidine intermediates via the reaction with orthoformates and morpholine followed by the triazine ring closing reaction of these intermediates with cyanamide. The conversion of 2-amino-4-phenylimidazole (1a) to the corresponding acetamidine (6) was achieved successfully using the reaction with triethyl orthoacetate and morpholine under microwave irradiation. The subsequent reaction of 6 with cyanamide led to the formation of a product identical to the one obtained from the MCR (Scheme 1). While the step-wise method excluded formation of compounds 2 and 3, a ring-closing reaction could still afford compounds 4 and/or 5a. The steric hindrance between the amino group and the phenyl ring in $\mathbf{4}$ would affect the thermodynamic stability of this potential product, therefore decreasing chances for its formation. Moreover, we could not exclude a rearrangement of $\mathbf{4}$ into 5 a under the reaction conditions. The rearrangement would proceed via intermediate 7 via the mechanism similar to the one reported ${ }^{21}$ for the rearrangement of another fused heterocycle with the amino-1,3,5-triazine ring. NOESY experiments performed on the product did not revealed any through-space interactions between the phenyl and methyl groups of the compound thus supporting assignment of structure 5a as the MCR product. Xray crystal structure determinations for analogous compounds $\mathbf{5 g}$ and 5p (vide infra) further confirmed the regioselective closure of the triazine ring to the more sterically accessible endocyclic nitrogen of the imidazole.

Using the optimized conditions from the model reaction, we explored the scope of our three-component reaction using a variety of trialkyl orthoesters and 4-aryl substituted 2-aminoimidazoles (1). The method was successfully applied for the synthesis of a library of 24 new 2-alkyl-4-amino-7-arylimidazo $[1,2-a][1,3,5]$ triazines (5) (Table 2). The reaction was found to proceed similarly for the full range of tested 2-aminoimidazoles accommodating aryl substituents in the position 4 of the imidazole ring. However, variations in the alkyl orthoester type 


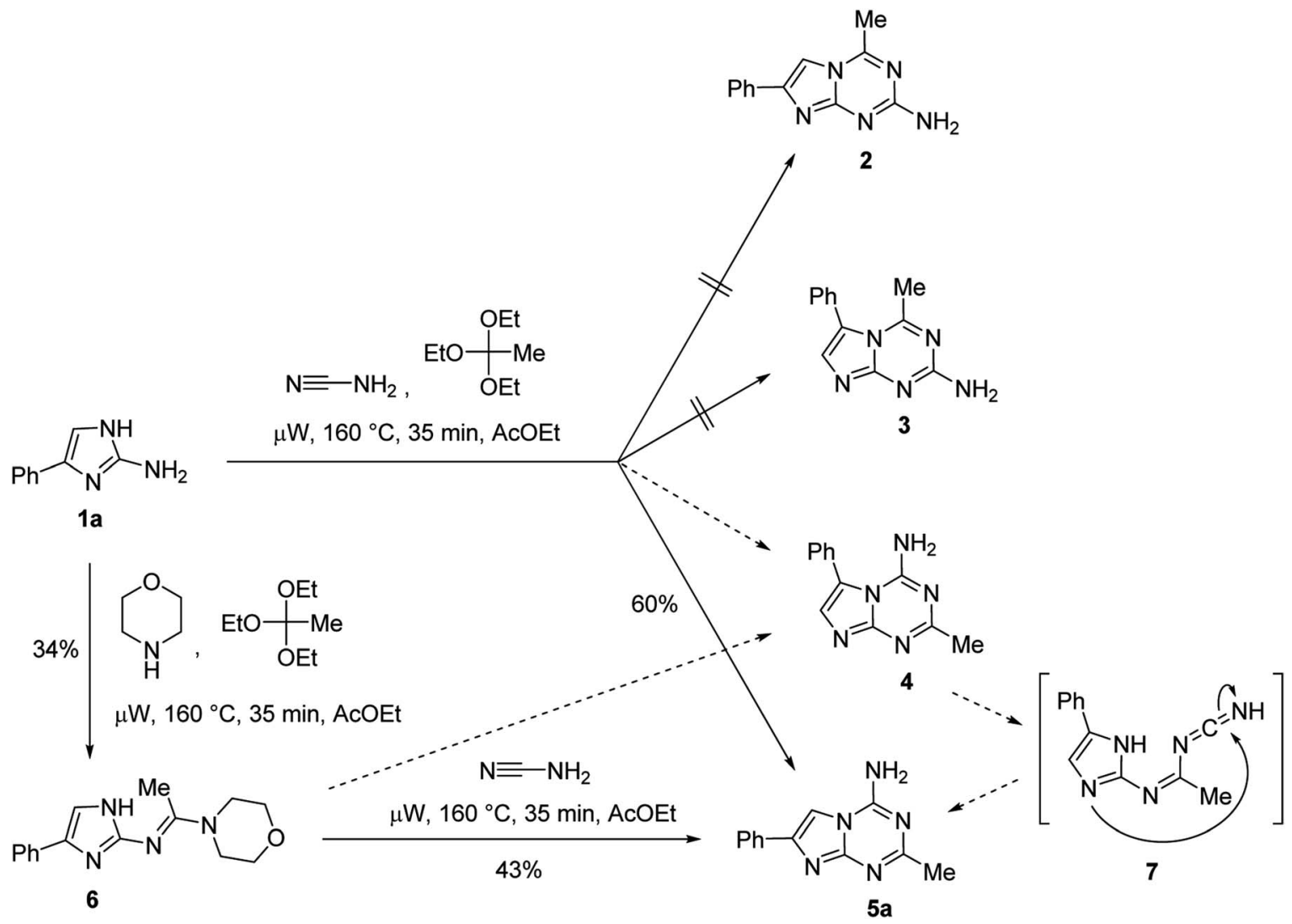

Scheme 1 Synthesis of 4-amino-2-methyl-7-phenylimidazo[1,2-a][1,3,5]triazine (5a).

affected product yields more significantly. In general, an increase in length of alkyl chain substituent on compound 5 led to a decrease of isolated yields. The yields of compounds $\mathbf{5 a}-\mathbf{f}$ and $\mathbf{5 g}-\mathbf{l}$ obtained from triethyl orthoacetate and triethyl orthopropionate, respectively, were substantially higher than those of $\mathbf{5 m}-\mathbf{r}$ and $\mathbf{5 s}-\mathbf{x}$ prepared using triethyl orthobutyrate and trimethyl orthovalerate. The decrease of yields in the homologous series of compounds $\mathbf{5}$ could be attributed to the relatively lower reactivity of long chain orthoesters used for their preparation. This assumption found a confirmation in the isolation of compounds 5 exclusively from all the reactions. No traces of other isomers 2-4 were detected. Besides products 5, only unreacted 2-aminoimidazoles (1) could be isolated from the reaction mixtures.

\section{X-ray crystallography}

Crystals were obtained for two representative compounds, namely $5 \mathrm{~g}$ and $\mathbf{5 p}$, and their crystal and molecular structures determined by X-ray crystallography. The molecular structure of $\mathbf{5 g}$ is shown in Fig. 1a and comprises an essentially planar ninemembered ring with the r.m.s. deviation of the fitted atoms being $0.0129 \AA$; the maximum deviations above and below the least-square plane are found for the C2 [0.0215(8) $\AA]$ and C9 [0.0232(9) $\AA]$ atoms, respectively; the N41 and C71 atoms lie, respectively, 0.0464(13) and 0.0413(13) A to the same side of the molecule.
Indeed, the entire molecule, with the exception of the C2bound ethyl group, is essentially co-planar as seen in the dihedral between the imidazo[1,2- $a][1,3,5]$ triazine and phenyl rings of $2.31(5)^{\circ}$. The ethyl group has an tanti-clinal (+ac) disposition with respect to the fused-ring system as seen in the torsion angle of $97.41(12)^{\circ}$ for $\mathrm{N} 1-\mathrm{C} 2-\mathrm{C} 21-\mathrm{C} 22$.

The crystallographic asymmetric of $\mathbf{5 p}$ comprises two independent molecules, illustrated in Fig. 1b. The molecules present features that differ from those described for $\mathbf{5 g}$. The r.m.s. of the nine atoms of the fused-ring system is $0.0247 \AA$ [0.0103 $\AA$ for the second independent molecule] with the N41 and C71 atoms lying 0.0263(15) and 0.0837(14) $\AA$ [0.0333(15) and $0.0113(14) \AA]$ to the same side of the plane. A twist in the molecule is evident as seen in the dihedral angle of $18.78(6)^{\circ}$ $\left[10.45(3)^{\circ}\right]$ between the rings. The C2-bound $n$-propyl groups have very similar conformations with the $\mathrm{C} 2-\mathrm{C} 21-\mathrm{C} 22-\mathrm{C} 23$ torsion angles being $-68.78(14)$ and $-72.27(13)^{\circ}$, indicating a - syn-clinal $(-\mathrm{sc})$ disposition in each case. The difference is that in the first independent molecule, the terminal methyl group is orientated away from the amino substituent whereas in the second molecule, it is orientated towards the amino group. An overlay diagram of the three molecules of $5 \mathrm{~g}$ and $5 \mathrm{p}$ is shown in Fig. 1c. The difference in the co-planarity of the aromatic residues of $\mathbf{5 g}$ and $\mathbf{5 p}$ is clearly evident, as are the different orientations of the $n$-propyl substituents in $\mathbf{5 p}$.

The molecular packing arrangements in the crystals of $\mathbf{5 g}$ and $5 p$ feature directional amino- $\mathrm{N}-\mathrm{H} \cdots \mathrm{N}$ (ring) hydrogen 
Table 2 Three-component synthesis of 2-alkyl-4-amino-7-arylimidazo[1,2-a][1,3,5]triazines (5) ${ }^{a}$

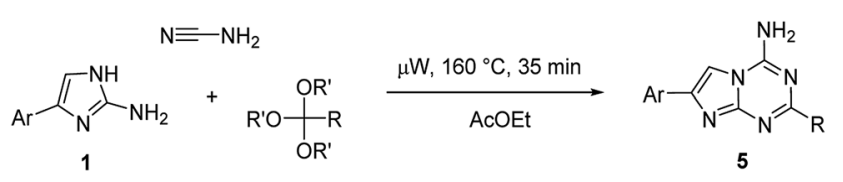

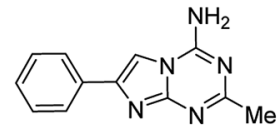

5 a, $60 \%$<smiles>Cc1nc(N)n2cc(-c3ccc(F)cc3)nc2n1</smiles>

5b, $60 \%$<smiles>Cc1nc(N)n2cc(-c3ccc(Cl)cc3)nc2n1</smiles>

$5 c, 30 \%$<smiles>Cc1ccc(-c2cn3c(N)nc(C)nc3n2)cc1</smiles>

5d, $60 \%$<smiles>COc1ccc(-c2cn3c(N)nc(C)nc3n2)cc1</smiles>

5e, $61 \%$

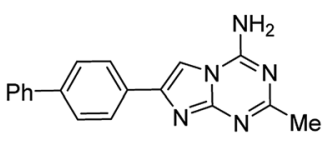

5f, $57 \%$

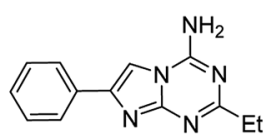

5g, $53 \%$<smiles>CCc1nc(N)n2cc(-c3ccc(F)cc3)nc2n1</smiles>

5h, $52 \%$<smiles>CCc1nc(N)n2cc(-c3ccc(Cl)cc3)nc2n1</smiles>

5i, $25 \%$<smiles>CCc1nc(N)n2cc(-c3ccc(C)cc3)nc2n1</smiles>

5j, $61 \%$<smiles>CCc1nc(N)n2cc(-c3ccc(OC)cc3)nc2n1</smiles>

5k, $57 \%$

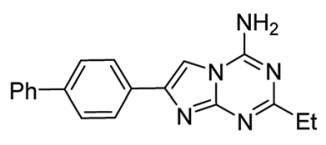

5I, $49 \%$

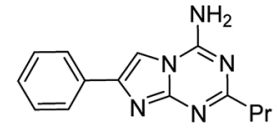

5m, $35 \%$<smiles>CCCc1nc(N)n2cc(-c3ccc(F)cc3)nc2n1</smiles>

5n, $36 \%$<smiles>CCCc1nc(N)n2cc(-c3ccc(Cl)cc3)nc2n1</smiles>

5o, $16 \%$<smiles>CCCc1nc(N)n2cc(-c3ccc(C)cc3)nc2n1</smiles>

5p, $50 \%$<smiles>CCCc1nc(N)n2cc(-c3ccc(OC)cc3)nc2n1</smiles>

$5 q, 52 \%$

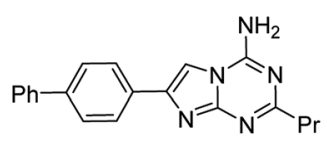

5r, $40 \%$

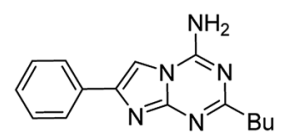

5s, $30 \%$<smiles>CC(C)Cc1nc(N)n2cc(-c3ccc(F)cc3)nc2n1</smiles>

5t, $29 \%$<smiles>CC(C)c1nc(N)n2cc(-c3ccc(Cl)cc3)nc2n1</smiles>

5u, $11 \%$<smiles>Cc1ccc(-c2cn3c(N)nc(C(C)C)nc3n2)cc1</smiles>

5v, 30\%<smiles>COc1ccc(-c2cn3c(N)nc(CC(C)C)nc3n2)cc1</smiles>

5w, $25 \%$

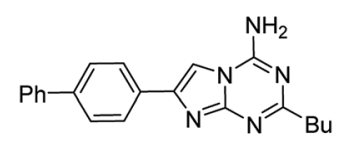

$5 x, 43 \%$

${ }^{a}$ The reaction was performed using a Discover SP CEM microwave synthesizer with $1 \mathrm{mmol}$ of 2-aminoimidazoles (1), $2.5 \mathrm{mmol}$ of trialkyl orthoesters and $2.5 \mathrm{mmol}$ of cyanamide at $160^{\circ} \mathrm{C}$ for $35 \mathrm{~min}$ in $2 \mathrm{~mL}$ of ethyl acetate.

bonding. In $\mathbf{5 g}$, centrosymmetrically-related molecules are connected via amino- $\mathrm{N}-\mathrm{H} \cdots \mathrm{N} 3$ (triazine) hydrogen bonds to form eight-membered $\{\cdots \mathrm{HNCN}\}_{2}$ synthons. The second amino$\mathrm{N}-\mathrm{H}$ hydrogen atom forms a hydrogen bond to the triazine-N1 atom. The result is a supramolecular layer parallel to $\left(\begin{array}{lll}1 & 0 & 0\end{array}\right)$ and with a zig-zag topology with the phenyl groups lying to either side, Fig. 2a. The layers stack along the $a$-axis with the primary interactions between them being of the type $\pi \cdots \pi$. The closest such interactions [3.4699(6) $\AA]$ occur between imidazo rings, and between phenyl rings [3.8142(7) $\AA$ ] . A view of the unit cell contents is shown in ESI Fig. S1 and geometric parameters characterizing the intermolecular interactions are given in the figure caption.

A supramolecular layer sustained by amino- $\mathrm{N}-\mathrm{H} \cdots \mathrm{N}$ (ring) hydrogen bonding is also found in the crystal of $5 \mathbf{p}$. The eightmembered $\{\cdots \mathrm{HNCN}\}_{2}$ synthons arising from amino- $\mathrm{N}-\mathrm{H} \cdots$ 
(a)

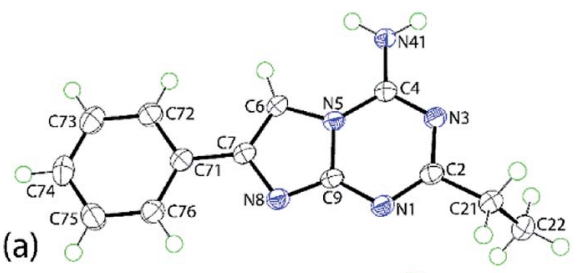

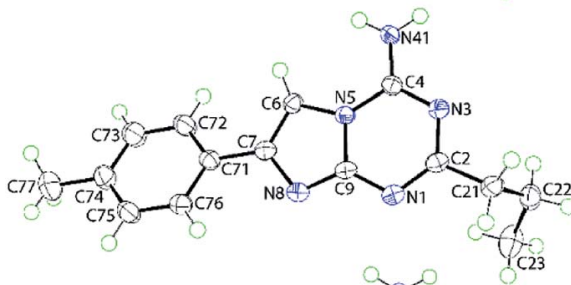

(c)

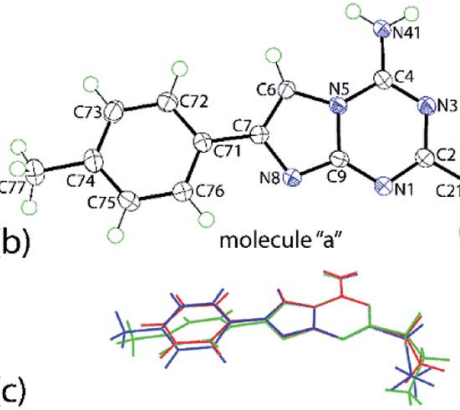

Fig. 1 (a) Molecular structure of $5 \mathrm{~g}$ and (b) molecular structures of the two independent molecules comprising the asymmetric unit of $5 p$, showing atom labelling scheme and $70 \%$ anisotropic displacement parameters. (c) An overlay diagram of $5 \mathrm{~g}$ (red image), the first independent molecule of $5 p$ (green) and the inverted second independent molecule of $5 p$ (blue). The molecules have been overlapped so the triazine rings are coincident.

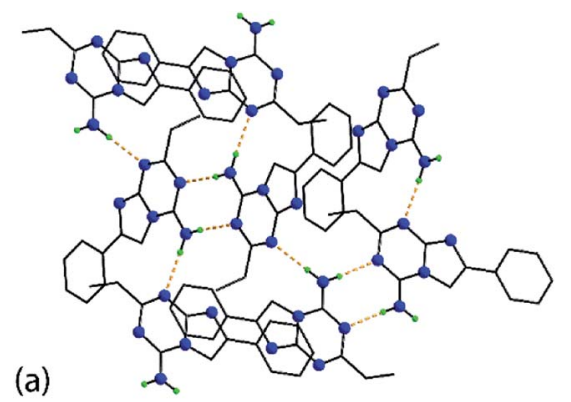

(b)

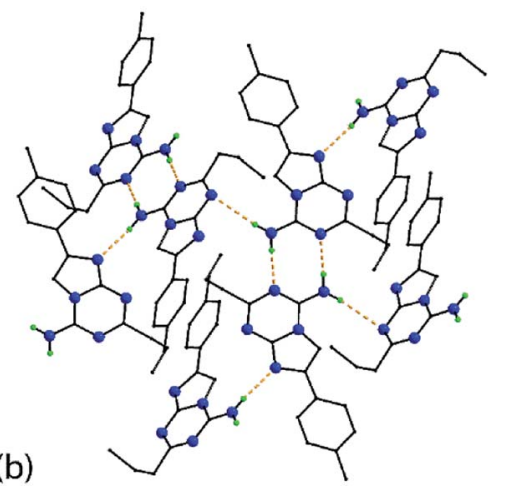

Fig. 2 Supramolecular layers sustained by amino- $\mathrm{N}-\mathrm{H} \cdots \mathrm{N}$ (ring) hydrogen bonding (see ESI Fig. S1 $\$$ ), shown as orange dashed lines, in the crystals of (a) $5 \mathrm{~g}$ and (b) $5 \mathrm{p}$. Non-participating hydrogen atoms have been omitted and the carbon atoms are represented as small spheres.
N3(triazine) hydrogen bonds is formed by each of the independent molecules which self-associate. For the first independent molecule, rather than an amino- $\mathrm{N}-\mathrm{H} \cdots \mathrm{N} 3$ (triazine) hydrogen bond as formed by the second independent molecule (and in the crystal of $\mathbf{5 g}$ ), an amino- $\mathrm{N}-\mathrm{H} \cdots \mathrm{N} 8$ (imidazo) hydrogen bond is formed instead; the latter pairs of interactions involve both independent molecules. As in 5g, the supramolecular layer, which is approximately parallel to $\left(\begin{array}{lll}1 & 0 & 1\end{array}\right)$, has a jagged topology with the 4-tolyl rings projecting to either side, Fig. $2 \mathrm{~b}$. The connections between layers to consolidate the three-dimensional architecture are of the type $\pi$ (imidazo) $\cdots$ $\pi($ imidazo) [3.5695(7) $\AA$ ] and $\pi$ (triazine) $\cdots \pi$ (phenyl) [3.8946(7) and 3.9224(7) $\AA$ ]. A view of the unit cell contents is shown in ESI Fig. S2 $\$$ with geometric parameters given in the chart caption.

\section{Conclusion}

In summary, we have successfully developed a three component reaction for the synthesis of novel 2-alkyl substituted 4-aminoimidazo[1,2- $a][1,3,5]$ triazines 5 using easily accessible starting materials and a simple, catalyst- and chromatography-free protocol. The distinct advantage of the proposed approach was found to be high chemo- and regio-selectivity with the exclusive formation of desired products in high purity.

\section{Experimental section}

\section{General information}

Melting points (uncorrected) were determined on a Stuart ${ }^{\mathrm{TM}}$ SMP40 automatic melting point apparatus. ${ }^{1} \mathrm{H}$ and ${ }^{13} \mathrm{C}$ NMR spectra were recorded on a Bruker Fourier 300 spectrometer (300 MHz) using DMSO- $d_{6}$ as a solvent and TMS as an internal reference. Microwave-assisted reactions were carried out in the closed vessel focused single mode using a Discover SP microwave synthesizer (CEM, USA) monitoring reaction temperature by equipped IR sensor. The model reaction was also carried out using Monowave 400 (Anton Paar, Austria) and Monowave 50 (Anton Paar, Austria) reactors.

General method for the synthesis of 4-amino-2-alkyl-7-arylimidazo[1,2-a][1,3,5]triazines (5) under microwave irradiation. The mixture of 2 -aminoimidazoles $(1,1 \mathrm{mmol})$, cyanamide $(105 \mathrm{mg}, 2.5 \mathrm{mmol})$ and trialkyl orthoesters $(2.5 \mathrm{mmol})$ in ethyl acetate $(2 \mathrm{~mL})$ were irradiated in a $10 \mathrm{~mL}$ seamless pressure vial using a microwave system operating at maximal microwave power up to $150 \mathrm{~W}$ (Discover SP, CEM) or $850 \mathrm{~W}$ (Monowave 400, Anton Paar) at $160{ }^{\circ} \mathrm{C}$ for $35 \mathrm{~min}$. After cooling, the precipitate was filtered, washed with ethyl acetate and recrystallised from appropriate solvents.

4-Amino-2-methyl-7-phenylimidazo[1,2-a][1,3,5]triazine (5a). Light yellow solid, yield $60 \%$, mp $303-305{ }^{\circ} \mathrm{C}(\mathrm{EtOH}) .{ }^{1} \mathrm{H}$ NMR (300 MHz, DMSO- $\left.d_{6}\right): \delta 2.38\left(3 \mathrm{H}, \mathrm{s}, \mathrm{CH}_{3}\right), 7.35\left(1 \mathrm{H}, \mathrm{t},{ }^{3} J=7.4 \mathrm{~Hz}\right.$, $\left.\mathrm{H}-4^{\prime}\right), 7.47\left(2 \mathrm{H}, \mathrm{t},{ }^{3} J=7.5 \mathrm{~Hz}, \mathrm{H}-3^{\prime}\right.$ and $\left.\mathrm{H}-5^{\prime}\right), 7.85\left(2 \mathrm{H}, \mathrm{d},{ }^{3} J=\right.$ $7.0 \mathrm{~Hz}, \mathrm{H}-2^{\prime}$ and $\left.\mathrm{H}-6^{\prime}\right), 8.15(1 \mathrm{H}, \mathrm{s}, \mathrm{H}-6), 8.35\left(2 \mathrm{H}, \mathrm{br} \mathrm{s}, \mathrm{NH}_{2}\right) .{ }^{13} \mathrm{C}$ NMR (75 MHz, DMSO- $\left.d_{6}\right): \delta 25.3\left(\mathrm{CH}_{3}\right), 101.7(\mathrm{C}-6), 125.2\left(\mathrm{C}-2^{\prime}\right.$ and C-6'), $127.9\left(\mathrm{C}-4^{\prime}\right), 128.7\left(\mathrm{C}-3^{\prime}\right.$ and $\left.\mathrm{C}-5^{\prime}\right), 133.3\left(\mathrm{C}-1^{\prime}\right), 143.2$ (C-7), 150.3 \& 150.6 (C-4 and C-8a), 164.7 (C-2). Anal. Calcd for 
$\mathrm{C}_{12} \mathrm{H}_{11} \mathrm{~N}_{5}: \mathrm{C}, 63.99 ; \mathrm{H}, 4.92 ; \mathrm{N}, 31.09$. Found: $\mathrm{C}, 63.82 ; \mathrm{H}$, $5.08 ; \mathrm{N}, 30.91$.

4-Amino-2-methyl-7-(4-fluorophenyl)imidazo[1,2-a][1,3,5]triazine (5b). White solid, yield $60 \%, \mathrm{mp}>350{ }^{\circ} \mathrm{C}($ EtOH $) .{ }^{1} \mathrm{H}$ NMR $(300$ $\left.\mathrm{MHz}, \mathrm{DMSO}-d_{6}\right): \delta 2.38\left(3 \mathrm{H}, \mathrm{s}, \mathrm{CH}_{3}\right), 7.30\left(2 \mathrm{H}, \mathrm{dd},{ }^{3} J_{\mathrm{HF}}=8.9 \mathrm{~Hz}\right.$, ${ }^{3} J_{\mathrm{HH}}=8.9 \mathrm{~Hz}, \mathrm{H}-3^{\prime}$ and $\left.\mathrm{H}-5^{\prime}\right), 7.87\left(2 \mathrm{H}, \mathrm{dd},{ }^{4} J_{\mathrm{HF}}=5.5 \mathrm{~Hz},{ }^{3} J_{\mathrm{HH}}=\right.$ $8.9 \mathrm{~Hz}, \mathrm{H}-2^{\prime}$ and $\left.\mathrm{H}^{-6} 6^{\prime}\right), 8.11$ (1H, s, H-6), 8.37 (2H, br s, $\left.\mathrm{NH}_{2}\right) .{ }^{13} \mathrm{C}$ NMR (75 MHz, DMSO- $\left.d_{6}\right): \delta 25.3\left(\mathrm{CH}_{3}\right), 101.5(\mathrm{C}-6), 115.7\left(\mathrm{~d},{ }^{2} J_{\mathrm{CF}}\right.$ $=21.7 \mathrm{~Hz}, \mathrm{C}-3^{\prime}$ and C-5'), $127.2\left(\mathrm{~d},{ }^{3} J_{\mathrm{CF}}=8.3 \mathrm{~Hz}, \mathrm{C}-2^{\prime}\right.$ and $\left.\mathrm{C}-6^{\prime}\right)$, $129.8\left(\mathrm{~d},{ }^{4} J_{\mathrm{CF}}=2.9 \mathrm{~Hz}, \mathrm{C}-\mathbf{1}^{\prime}\right), 142.3(\mathrm{C}-7), 150.4 \& 150.6(\mathrm{C}-4$ and C-8a), 161.9 (d, $\left.{ }^{1} J_{\mathrm{CF}}=244.7 \mathrm{~Hz}, \mathrm{C}-4^{\prime}\right), 164.8$ (C-2). Anal. Calcd for $\mathrm{C}_{12} \mathrm{H}_{10} \mathrm{FN}_{5}$ : C, 59.25; H, 4.14; N, 28.79. Found: C, 59.02; H, $4.30 ; \mathrm{N}, 28.63$.

4-Amino-2-methyl-7-(4-chlorophenyl)imidazo[1,2-a][1,3,5]triazine (5c). Brown solid, yield 30\%, mp $>350{ }^{\circ} \mathrm{C}(\mathrm{EtOH}) .{ }^{1} \mathrm{H}$ NMR $(300$ MHz, DMSO- $\left.d_{6}\right): \delta 2.37\left(3 \mathrm{H}, \mathrm{s}, \mathrm{CH}_{3}\right), 7.53\left(2 \mathrm{H}, \mathrm{d},{ }^{3} J=8.6 \mathrm{~Hz}, \mathrm{H}-3^{\prime}\right.$ and $\left.\mathrm{H}-5^{\prime}\right), 7.9\left(2 \mathrm{H}, \mathrm{d},{ }^{3} J=8.58 \mathrm{~Hz}, \mathrm{H}-2^{\prime}\right.$ and $\left.\mathrm{H}-6^{\prime}\right), 8.16(1 \mathrm{H}, \mathrm{s}, \mathrm{H}-$ 6), $8.38\left(2 \mathrm{H}\right.$, br s, $\left.\mathrm{NH}_{2}\right) .{ }^{13} \mathrm{C}$ NMR (75 MHz, DMSO- $\left.d_{6}\right): \delta 25.3$ $\left(\mathrm{CH}_{3}\right), 102.1$ (C-6), 126.9 (C-2' and C-6'), 128.8 (C-3' and C-5'), $132.1\left(\mathrm{C}-1^{\prime}\right), 132.3$ (C-4'), 142.1 (C-7), 150.6 \& 150.4 (C-4 and C-8a), 164.9 (C-2). Anal. Calcd for $\mathrm{C}_{12} \mathrm{H}_{10} \mathrm{ClN}_{5}$ : C, 55.50; H, 3.88; N, 26.97. Found: 55.42; H, 4.01; N, 26.75.

4-Amino-2-methyl-7-(4-methylphenyl)imidazo[1,2-a][1,3,5] triazine (5d). White solid, yield $60 \%, \mathrm{mp}>350{ }^{\circ} \mathrm{C}(\mathrm{EtOH}) .{ }^{1} \mathrm{H}$ NMR (300 MHz, DMSO- $\left.d_{6}\right): \delta 2.34\left(3 \mathrm{H}, \mathrm{s}, \mathrm{CH}_{3}\right), 2.37\left(3 \mathrm{H}, \mathrm{s}, \mathrm{CH}_{3}\right)$, $7.27\left(2 \mathrm{H}, \mathrm{d},{ }^{3} J=7.9 \mathrm{~Hz}, \mathrm{H}-3^{\prime}\right.$ and $\left.\mathrm{H}-5^{\prime}\right), 7.73\left(2 \mathrm{H}, \mathrm{d},{ }^{3} J=8.1 \mathrm{~Hz}\right.$, H-2' and H-6'), 8.09 (1H, s, H-6), $8.30\left(2 \mathrm{H}\right.$, br s, $\left.\mathrm{NH}_{2}\right) .{ }^{13} \mathrm{C}$ NMR (75 MHz, DMSO- $\left.d_{6}\right): \delta 20.8\left(\mathrm{CH}_{3}\right), 25.3\left(\mathrm{CH}_{3}\right), 101.1(\mathrm{C}-6), 125.2$ (C-2' and C-6'), 129.3 (C-3' and C-5'), $130.5\left(\mathrm{C}-1^{\prime}\right), 137.3\left(\mathrm{C}-4^{\prime}\right)$, 143.4 (C-7), 150.2 \& 150.5 (C-4 and C-8a), 164.5 (C-2). Anal. Calcd for $\mathrm{C}_{13} \mathrm{H}_{13} \mathrm{~N}_{5}$ : C, 65.25; H, 5.48; N, 29.27. Found: 65.18; $\mathrm{H}$, $5.60 ; \mathrm{N}, 29.18$.

4-Amino-2-methyl-7-(4-methoxyphenyl)imidazo[1,2-a][1,3,5]triazine (5e). Light yellow solid, yield 61\%, mp 338-339 ${ }^{\circ} \mathrm{C}(\mathrm{EtOH}) .{ }^{1} \mathrm{H}$ NMR (300 MHz, DMSO- $\left.d_{6}\right): \delta 2.37\left(3 \mathrm{H}, \mathrm{s}, \mathrm{CH}_{3}\right), 3.80\left(3 \mathrm{H}, \mathrm{s}, \mathrm{OCH}_{3}\right), 7.03$ $\left(2 \mathrm{H}, \mathrm{d},{ }^{3} J=8.9 \mathrm{~Hz}, \mathrm{H}-3^{\prime}\right.$ and $\left.\mathrm{H}-5^{\prime}\right), 7.77\left(2 \mathrm{H}, \mathrm{d},{ }^{3} J=8.9 \mathrm{~Hz}, \mathrm{H}-2^{\prime}\right.$ and H-6'), 8.02 (1H, s, H-6), 8.29 (2H, br s, $\left.\mathrm{NH}_{2}\right) .{ }^{13} \mathrm{C}$ NMR $(75 \mathrm{MHz}$, DMSO- $\left.d_{6}\right): \delta 25.3\left(\mathrm{CH}_{3}\right), 55.1\left(\mathrm{OCH}_{3}\right), 100.4(\mathrm{C}-6), 114.2\left(\mathrm{C}-3^{\prime}\right.$ and C$\left.5^{\prime}\right), 125.8\left(\mathrm{C}-1^{\prime}\right), 126.6\left(\mathrm{C}-2^{\prime}\right.$ and C-6 $\left.{ }^{\prime}\right), 143.3(\mathrm{C}-7), 150.3 \& 150.5(\mathrm{C}-4$ and C-8a), 159.2 (C-4'), 164.4 (C-2). Anal. Calcd for $\mathrm{C}_{13} \mathrm{H}_{13} \mathrm{~N}_{5} \mathrm{O}: \mathrm{C}$, 61.17; H, 5.13; N, 27.43. Found: C, 61.05; H, 5.23; N, 27.32.

4-Amino-2-methyl-7-(4-biphenyl)imidazo[1,2-a][1,3,5]triazine (5f). Light brown solid, yield 57\%, mp > $350{ }^{\circ} \mathrm{C}$ (DMF). ${ }^{1} \mathrm{H}$ NMR $(300$ MHz, DMSO- $\left.d_{6}\right): \delta 2.38\left(3 \mathrm{H}, \mathrm{s}, \mathrm{CH}_{3}\right), 7.38\left(1 \mathrm{H}, \mathrm{t},{ }^{3} J=7.3 \mathrm{~Hz}, \mathrm{H}-4^{\prime \prime}\right)$, $7.49\left(2 \mathrm{H}, \mathrm{t},{ }^{3} J=7.5 \mathrm{~Hz}, \mathrm{H}-3^{\prime \prime}\right.$ and $\left.\mathrm{H}-5^{\prime \prime}\right), 7.73\left(2 \mathrm{H}, \mathrm{d},{ }^{3} J=7.1 \mathrm{~Hz}, \mathrm{H}-\right.$ $2^{\prime \prime}$ and $\left.\mathrm{H}-6^{\prime \prime}\right), 7.79\left(2 \mathrm{H}, \mathrm{d},{ }^{3} \mathrm{~J}=8.4 \mathrm{~Hz}, \mathrm{H}-3^{\prime}\right.$ and $\left.\mathrm{H}-5^{\prime}\right), 7.94\left(2 \mathrm{H}, \mathrm{d},{ }^{3} J\right.$ $=8.4 \mathrm{~Hz}, \mathrm{H}-2^{\prime}$ and $\left.\mathrm{H}-6^{\prime}\right), 8.20(1 \mathrm{H}, \mathrm{s}, \mathrm{H}-6), 8.36\left(2 \mathrm{H}, \mathrm{br} \mathrm{s}, \mathrm{NH}_{2}\right) \cdot{ }^{13} \mathrm{C}$ NMR (75 MHz, DMSO- $\left.d_{6}\right): \delta 25.3\left(\mathrm{CH}_{3}\right), 101.8(\mathrm{C}-6), 125.8\left(\mathrm{C}-3^{\prime}\right.$ and $\left.\mathrm{C}-5^{\prime}\right), 126.4\left(\mathrm{C}-3^{\prime \prime}\right.$ and $\left.\mathrm{C}-5^{\prime \prime}\right), 127.0\left(\mathrm{C}-2^{\prime}\right.$ and $\left.\mathrm{C}-6^{\prime}\right), 127.4\left(\mathrm{C}-4^{\prime \prime}\right)$, $128.9\left(\mathrm{C}-2^{\prime \prime}\right.$ and C-6"), $132.4\left(\mathrm{C}-1^{\prime}\right), 139.5\left(\mathrm{C}-4^{\prime}\right.$ and C-1" $), 142.9$ (C7), 150.4 \& 150.6 (C-4 and C-8a), 164.7 (C-2). Anal. Calcd for $\mathrm{C}_{18} \mathrm{H}_{15} \mathrm{~N}_{5}$ : C, 71.74; H, 5.02; N, 23.24. Found: C, 71.68; H, 5.11; N, 23.10.

4-Amino-2-ethyl-7-phenylimidazo[1,2-a][1,3,5]triazine

(5g). Light yellow solid, yield 53\%, mp 299-301 ${ }^{\circ} \mathrm{C}$ (EtOH). ${ }^{1} \mathrm{H}$ NMR (300 MHz, DMSO- $\left.d_{6}\right): \delta 1.25\left(3 \mathrm{H}, \mathrm{t},{ }^{3} J=7.6 \mathrm{~Hz}, \mathrm{CH}_{3}\right), 2.66(2 \mathrm{H}, \mathrm{q}$, $\left.{ }^{3} J=7.5 \mathrm{~Hz}, \mathrm{CH}_{2}\right), 7.35\left(1 \mathrm{H}, \mathrm{t},{ }^{3} J=7.4 \mathrm{~Hz}, \mathrm{H}-4^{\prime}\right), 7.47\left(2 \mathrm{H}, \mathrm{t},{ }^{3} J=\right.$
$7.5 \mathrm{~Hz}, \mathrm{H}-3^{\prime}$ and $\left.\mathrm{H}-5^{\prime}\right), 7.86\left(2 \mathrm{H}, \mathrm{d},{ }^{3} J=7.0 \mathrm{~Hz}, \mathrm{H}-2^{\prime}\right.$ and $\left.\mathrm{H}-6^{\prime}\right)$, $8.16(1 \mathrm{H}, \mathrm{s}, \mathrm{H}-6), 8.36$ (2H, br s, $\left.\mathrm{NH}_{2}\right) ;{ }^{13} \mathrm{C}$ NMR (75 MHz, DMSO$\left.d_{6}\right): \delta 11.7\left(\mathrm{CH}_{3}\right), 31.5\left(\mathrm{CH}_{2}\right), 101.7(\mathrm{C}-6), 125.2\left(\mathrm{C}-2^{\prime}\right.$ and $\left.\mathrm{C}-6^{\prime}\right)$, $127.9\left(\mathrm{C}-4^{\prime}\right), 128.7\left(\mathrm{C}-3^{\prime}\right.$ and $\left.\mathrm{C}-5^{\prime}\right), 133.3\left(\mathrm{C}-1^{\prime}\right), 143.3(\mathrm{C}-7), 150.4$ \& 150.8 (C-4 and C-8a), 168.6 (C-2). Anal. Calcd for $\mathrm{C}_{13} \mathrm{H}_{13} \mathrm{~N}_{5}$ : C, 65.25; H, 5.48; N, 29.27. Found: C, 65.22; H, 5.52; N, 29.12.

4-Amino-2-ethyl-7-(4-fluorophenyl)imidazo[1,2-a][1,3,5]triazine (5h). White solid, yield 52\%, mp 339-340 ${ }^{\circ} \mathrm{C}(\mathrm{EtOH}) .{ }^{1} \mathrm{H}$ NMR (300 MHz, DMSO- $\left.d_{6}\right): \delta 1.25\left(3 \mathrm{H}, \mathrm{t},{ }^{3} J=7.6 \mathrm{~Hz}, \mathrm{CH}_{3}\right), 2.65(2 \mathrm{H}$, $\left.\mathrm{q},{ }^{3} J=7.5 \mathrm{~Hz}, \mathrm{CH}_{2}\right), 7.31\left(2 \mathrm{H}, \mathrm{dd},{ }^{3} J_{\mathrm{HF}}=8.9 \mathrm{~Hz},{ }^{3} J_{\mathrm{HH}}=8.9 \mathrm{~Hz}\right.$, $\mathrm{H}-3^{\prime}$ and $\left.\mathrm{H}-5^{\prime}\right), 7.88\left(2 \mathrm{H}, \mathrm{dd},{ }^{4} J_{\mathrm{HF}}=5.5 \mathrm{~Hz},{ }^{3} J_{\mathrm{HH}}=8.9 \mathrm{~Hz}, \mathrm{H}-2^{\prime}\right.$ and $\left.\mathrm{H}-6^{\prime}\right), 8.12$ (1H, s, H-6), $8.35\left(2 \mathrm{H}, \mathrm{br} \mathrm{s}, \mathrm{NH}_{2}\right) .{ }^{13} \mathrm{C} \mathrm{NMR}(75$ MHz, DMSO- $\left.d_{6}\right): \delta 11.7\left(\mathrm{CH}_{3}\right), 31.5\left(\mathrm{CH}_{2}\right), 101.5(\mathrm{C}-6), 115.7$ (d, ${ }^{2} J_{\mathrm{CF}}=21.8 \mathrm{~Hz}, \mathrm{C}-3^{\prime}$ and C-5 $\left.{ }^{\prime}\right), 127.2\left(\mathrm{~d},{ }^{3} J_{\mathrm{CF}}=8.3 \mathrm{~Hz}, \mathrm{C}-2^{\prime}\right.$ and C$\left.6^{\prime}\right), 129.9\left(\mathrm{~d},{ }^{4} J_{\mathrm{CF}}=3.0 \mathrm{~Hz}, \mathrm{C}-1^{\prime}\right), 142.4(\mathrm{C}-7), 150.4 \& 150.8(\mathrm{C}-4$ and C-8a), 161.9 (d, $\left.{ }^{1} J_{\mathrm{CF}}=245.1 \mathrm{~Hz}, \mathrm{C}-4^{\prime}\right), 168.7$ (C-2). Anal. Calcd for $\mathrm{C}_{13} \mathrm{H}_{12} \mathrm{FN}_{5}: \mathrm{C}, 60.69 ; \mathrm{H}, 4.70 ; \mathrm{N}, 27.22$. Found: $\mathrm{C}$, $60.62 ; \mathrm{H}, 4.83 ; \mathrm{N}, 27.11$.

4-Amino-2-ethyl-7-(4-chlorophenyl)imidazo[1,2-a][1,3,5]triazine (5i). Brown solid, yield 25\%, mp 340-342 ${ }^{\circ} \mathrm{C}(\mathrm{EtOH}) .{ }^{1} \mathrm{H}$ NMR (300 MHz, DMSO- $\left.d_{6}\right): \delta 1.24\left(3 \mathrm{H}, \mathrm{t},{ }^{3} J=7.6 \mathrm{~Hz}, \mathrm{CH}_{3}\right), 2.65(2 \mathrm{H}, \mathrm{q}$, $\left.{ }^{3} J=7.5 \mathrm{~Hz}, \mathrm{CH}_{2}\right), 7.53\left(2 \mathrm{H}, \mathrm{d},{ }^{3} J=8.7 \mathrm{~Hz}, \mathrm{H}-3^{\prime}\right.$ and $\left.\mathrm{H}-5^{\prime}\right), 7.85$ $\left(2 \mathrm{H}, \mathrm{d},{ }^{3} J=8.7 \mathrm{~Hz}, \mathrm{H}-2^{\prime}\right.$ and $\left.\mathrm{H}-6^{\prime}\right), 8.17(1 \mathrm{H}, \mathrm{s}, \mathrm{H}-6), 8.36(2 \mathrm{H}$, br s, $\left.\mathrm{NH}_{2}\right) \cdot{ }^{13} \mathrm{C}$ NMR (75 MHz, DMSO- $\left.d_{6}\right): \delta 11.7\left(\mathrm{CH}_{3}\right), 31.5$ $\left(\mathrm{CH}_{2}\right.$ ), 102.1 (C-6), 126.9 (C-2' and C-6'), 128.8 (C-3' and C-5'), $132.2\left(\mathrm{C}-1^{\prime}\right), 132.3$ (C-4'), 142.1 (C-7), $150.5 \& 150.8$ (C-4 and C8a), 168.8 (C-2). Anal. Calcd for $\mathrm{C}_{13} \mathrm{H}_{12} \mathrm{ClN}_{5}$ : C, 57.04; $\mathrm{H}$, 4.42; N, 25.59. Found: C, 56.97; H, 4.50; N, 25.49.

4-Amino-2-ethyl-7-(4-methyphenyl)imidazo[1,2-a][1,3,5]triazine (5j). Light yellow solid, yield 61\%, mp $>350{ }^{\circ} \mathrm{C}$ (EtOH). ${ }^{1} \mathrm{H}$ NMR $\left(300 \mathrm{MHz}, \mathrm{DMSO}-d_{6}\right): \delta 1.25\left(3 \mathrm{H}, \mathrm{t},{ }^{3} J=7.6 \mathrm{~Hz}, \mathrm{CH}_{3}\right), 2.34(3 \mathrm{H}, \mathrm{s}$, $\left.\mathrm{CH}_{3}\right), 2.65\left(2 \mathrm{H}, \mathrm{q},{ }^{3} J=7.5 \mathrm{~Hz}, \mathrm{CH}_{2}\right), 7.27\left(2 \mathrm{H}, \mathrm{d},{ }^{3} J=8.0 \mathrm{~Hz}, \mathrm{H}-3\right.$ and $\mathrm{H}-5), 7.75\left(2 \mathrm{H}, \mathrm{d},{ }^{3} J=8.1 \mathrm{~Hz}, \mathrm{H}-2^{\prime}\right.$ and $\left.\mathrm{H}-6^{\prime}\right), 8.10(1 \mathrm{H}, \mathrm{s}, \mathrm{H}-$ 6), $8.31\left(2 \mathrm{H}\right.$, br s, $\left.\mathrm{NH}_{2}\right) .{ }^{13} \mathrm{C} \mathrm{NMR}\left(75 \mathrm{MHz}\right.$, DMSO- $\left.d_{6}\right): \delta 11.7$ $\left(\mathrm{CH}_{3}\right), 20.8\left(\mathrm{CH}_{3}\right), 31.5\left(\mathrm{CH}_{2}\right), 101.2(\mathrm{C}-6), 125.2\left(\mathrm{C}-2^{\prime}\right.$ and $\left.\mathrm{C}-6^{\prime}\right)$, $129.3\left(\mathrm{C}-3^{\prime}\right.$ and $\left.\mathrm{C}-5^{\prime}\right), 130.6\left(\mathrm{C}-1^{\prime}\right), 137.3\left(\mathrm{C}-4^{\prime}\right), 143.5(\mathrm{C}-7), 150.3$ \& 150.7 (C-4 and C-8a), 168.5 (C-2). Anal. Calcd for $\mathrm{C}_{14} \mathrm{H}_{15} \mathrm{~N}_{5}: \mathrm{C}$, 66.38; H, 5.97; N, 27.65. Found: C, 66.33; H, 6.08; N, 27.58.

4-Amino-2-ethyl-7-(4-methoxyphenyl)imidazo[1,2-a][1,3,5]triazine (5k). White solid, yield 57\%, mp $328-330{ }^{\circ} \mathrm{C}(\mathrm{EtOH}) .{ }^{1} \mathrm{H}$ NMR $(300$ MHz, DMSO- $\left.d_{6}\right): \delta 1.24\left(3 \mathrm{H}, \mathrm{t},{ }^{3} J=7.6 \mathrm{~Hz}, \mathrm{CH}_{3}\right), 2.64\left(2 \mathrm{H}, \mathrm{q},{ }^{3} J=\right.$ $\left.7.6 \mathrm{~Hz}, \mathrm{CH}_{2}\right), 3.80\left(3 \mathrm{H}, \mathrm{s}, \mathrm{OCH}_{3}\right), 7.04\left(2 \mathrm{H}, \mathrm{d},{ }^{3} \mathrm{~J}=8.9 \mathrm{~Hz}, \mathrm{H}-3^{\prime}\right.$ and $\left.\mathrm{H}-5^{\prime}\right), 7.78\left(2 \mathrm{H}, \mathrm{d},{ }^{3} J=8.9 \mathrm{~Hz}, \mathrm{H}-2^{\prime}\right.$ and $\left.\mathrm{H}-6^{\prime}\right), 8.02(1 \mathrm{H}, \mathrm{s}, \mathrm{H}-6)$, $8.29\left(2 \mathrm{H}\right.$, br s, $\left.\mathrm{NH}_{2}\right) .{ }^{13} \mathrm{C}$ NMR (75 MHz, DMSO- $\left.d_{6}\right): \delta 11.7$ $\left(\mathrm{CH}_{3}\right), 31.4\left(\mathrm{CH}_{2}\right), 100.4(\mathrm{C}-6), 114.2\left(\mathrm{C}-2^{\prime}\right.$ and $\left.\mathrm{C}-6^{\prime}\right), 125.9\left(\mathrm{C}-4^{\prime}\right)$, 126.6 (C-3' and C-5'), 143.4 (C-7), $150.3 \& 150.6$ (C-4 and C-8a), $159.2\left(\mathrm{C}-1^{\prime}\right), 168.3$ (C-2). Anal. Calcd for $\mathrm{C}_{14} \mathrm{H}_{15} \mathrm{~N}_{5} \mathrm{O}: \mathrm{C}, 62.44 ; \mathrm{H}$, 5.61; N, 26.01. Found: C, 62.38; H, 5.74; N, 25.90.

4-Amino-2-ethyl-7-(4-biphenyl)imidazo[1,2-a][1,3,5]triazine (5l). White solid, yield 49\%, mp 348-350 ${ }^{\circ} \mathrm{C}$ (DMF). ${ }^{1} \mathrm{H}$ NMR (300 MHz, DMSO- $\left.d_{6}\right): \delta 1.26\left(3 \mathrm{H}, \mathrm{t},{ }^{3} J=7.6 \mathrm{~Hz}, \mathrm{CH}_{3}\right), 2.66\left(2 \mathrm{H}, \mathrm{q},{ }^{3} J=\right.$ $7.6 \mathrm{~Hz} \mathrm{CH}$ ), $7.38\left(1 \mathrm{H}, \mathrm{t},{ }^{3} J=7.3 \mathrm{~Hz}, \mathrm{H}-4^{\prime \prime}\right), 7.49\left(2 \mathrm{H}, \mathrm{t},{ }^{3} \mathrm{~J}=\right.$ $7.5 \mathrm{~Hz}, \mathrm{H}-3^{\prime \prime}$ and $\left.\mathrm{H}-5^{\prime \prime}\right), 7.73\left(2 \mathrm{H}, \mathrm{d},{ }^{3} J=7.2 \mathrm{~Hz}, \mathrm{H}-2^{\prime \prime}\right.$ and $\left.\mathrm{H}-6^{\prime \prime}\right)$, $7.79\left(2 \mathrm{H}, \mathrm{d},{ }^{3} J=8.4 \mathrm{~Hz}, \mathrm{H}-3^{\prime}\right.$ and $\left.\mathrm{H}-5^{\prime}\right), 7.95\left(2 \mathrm{H}, \mathrm{d},{ }^{3} J=8.3 \mathrm{~Hz}\right.$, $\mathrm{H}-2^{\prime}$ and $\left.\mathrm{H}-6^{\prime}\right), 8.21$ (1H, s, H-6), $8.36\left(2 \mathrm{H}\right.$, br s, $\left.\mathrm{NH}_{2}\right) .{ }^{13} \mathrm{C} \mathrm{NMR}$ (75 MHz, DMSO- $\left.d_{6}\right): \delta 11.7\left(\mathrm{CH}_{3}\right), 31.5\left(\mathrm{CH}_{2}\right), 101.9(\mathrm{C}-6), 125.8$ (C-3' and C-5'), 126.4 (C-3" and C-5"), 127.0 (C-2' and C-6'), 127.4 
$\left(\mathrm{C}-4^{\prime \prime}\right), 128.9\left(\mathrm{C}-2^{\prime \prime}\right.$ and $\left.\mathrm{C}-6^{\prime \prime}\right), 132.4\left(\mathrm{C}-1^{\prime}\right), 139.5 \& 139.6\left(\mathrm{C}-4^{\prime}\right.$ and C-1"), 143.0 (C-7), 150.5 \& 150.8 (C-4 and C-8a), 168.7 (C-2). Anal. Calcd for $\mathrm{C}_{19} \mathrm{H}_{17} \mathrm{~N}_{5}$ : C, 72.36; H, 5.43; N, 22.21. Found: C, 72.31; $\mathrm{H}, 5.52 ; \mathrm{N}, 22.14$.

4-Amino-2-propyl-7-phenylimidazo[1,2-a][1,3,5]triazine (5m). Light yellow solid, yield $35 \%$, mp 272-274 ${ }^{\circ} \mathrm{C}$ (EtOH). ${ }^{1} \mathrm{H}$ NMR (300 MHz, DMSO- $\left.d_{6}\right): \delta 0.94\left(3 \mathrm{H}, \mathrm{t},{ }^{3} J=7.4 \mathrm{~Hz}, \mathrm{CH}_{3}\right), 1.77(2 \mathrm{H}$, $\left.\mathrm{m},{ }^{3} J=7.4 \mathrm{~Hz}, \mathrm{CH}_{2}\right), 2.60\left(2 \mathrm{H}, \mathrm{t},{ }^{3} J=7.4 \mathrm{~Hz}, \mathrm{CH}_{2}\right), 7.35\left(1 \mathrm{H}, \mathrm{t},{ }^{3} J\right.$ $\left.=7.3 \mathrm{~Hz}, \mathrm{H}-4^{\prime}\right), 7.47\left(2 \mathrm{H}, \mathrm{t},{ }^{3} J=7.5 \mathrm{~Hz}, \mathrm{H}-3^{\prime}\right.$ and $\left.\mathrm{H}-5^{\prime}\right), 7.85(2 \mathrm{H}$, $\mathrm{d},{ }^{3} J=7.1 \mathrm{~Hz}, \mathrm{H}-2^{\prime}$ and $\left.\mathrm{H}-6^{\prime}\right), 8.15(1 \mathrm{H}, \mathrm{s}, \mathrm{H}-6), 8.35$ (2H, br s, $\left.\mathrm{NH}_{2}\right) \cdot{ }^{13} \mathrm{C}$ NMR (75 MHz, DMSO- $\left.d_{6}\right): \delta 13.6\left(\mathrm{CH}_{3}\right), 20.4\left(\mathrm{CH}_{2}\right)$, $40.2\left(\mathrm{CH}_{2}\right), 101.7$ (C-6), $125.2\left(\mathrm{C}-2^{\prime}\right.$ and C-6'), $127.9\left(\mathrm{C}-4^{\prime}\right), 128.7$ $\left(\mathrm{C}-3^{\prime}\right.$ and $\left.\mathrm{C}-5^{\prime}\right), 133.3\left(\mathrm{C}-1^{\prime}\right), 143.3(\mathrm{C}-7), 150.3 \& 150.8(\mathrm{C}-4$ and $\mathrm{C}-$ 8a), 167.6 (C-2). Anal. Calcd for $\mathrm{C}_{14} \mathrm{H}_{15} \mathrm{~N}_{5}$ : C, 66.38; H, 5.97; N, 27.65. Found: C, 66.31; H, 6.02; N, 27.60.

4-Amino-2-propyl-7-(4-fluorophenyl)imidazo[1,2-a][1,3,5]triazine (5n). White solid, yield 36\%, mp 290-292 ${ }^{\circ} \mathrm{C}$ (EtOH). ${ }^{1} \mathrm{H}$ NMR $\left(300 \mathrm{MHz}, \mathrm{DMSO}-d_{6}\right): \delta 0.94\left(3 \mathrm{H}, \mathrm{t},{ }^{3} J=7.4 \mathrm{~Hz}, \mathrm{CH}_{3}\right), 1.76(2 \mathrm{H}$, $\left.\mathrm{m},{ }^{3} \mathrm{~J}=7.4 \mathrm{~Hz}, \mathrm{CH}_{2}\right), 2.60\left(2 \mathrm{H}, \mathrm{t},{ }^{3} J=7.4 \mathrm{~Hz}, \mathrm{CH}_{2}\right), 7.31(2 \mathrm{H}, \mathrm{dd}$, ${ }^{3} J_{\mathrm{HF}}=8.9 \mathrm{~Hz},{ }^{3} J_{\mathrm{HH}}=8.9 \mathrm{~Hz}, \mathrm{H}-3^{\prime}$ and $\left.\mathrm{H}-5^{\prime}\right), 7.87\left(2 \mathrm{H}, \mathrm{dd},{ }^{4} J_{\mathrm{HF}}=\right.$ $5.5 \mathrm{~Hz},{ }^{3} J_{\mathrm{HH}}=8.9 \mathrm{~Hz}, \mathrm{H}-2^{\prime}$ and $\left.\mathrm{H}^{\prime} 6^{\prime}\right), 8.11(1 \mathrm{H}, \mathrm{s}, \mathrm{H}-6), 8.34(2 \mathrm{H}$, br s, $\left.\mathrm{NH}_{2}\right) \cdot{ }^{13} \mathrm{C}$ NMR (75 MHz, DMSO- $\left.d_{6}\right): \delta 13.6\left(\mathrm{CH}_{3}\right), 20.4$ $\left(\mathrm{CH}_{2}\right), 40.2\left(\mathrm{CH}_{2}\right), 101.5(\mathrm{C}-6), 115.7\left(\mathrm{~d},{ }^{2} J_{\mathrm{CF}}=21.7 \mathrm{~Hz}, \mathrm{C}-3^{\prime}\right.$ and C-5'), $127.2\left(\mathrm{~d},{ }^{3} J_{\mathrm{CF}}=8.3 \mathrm{~Hz}, \mathrm{C}-2^{\prime}\right.$ and $\left.\mathrm{C}-6^{\prime}\right), 129.9\left(\mathrm{~d},{ }^{4} J_{\mathrm{CF}}=\right.$ $3.5 \mathrm{~Hz}, \mathrm{C}-1^{\prime}$ ), 142.4 (C-7), 150.4 \& 150.8 (C-4 and C-8a), 161.9 (d, ${ }^{1} J_{\mathrm{CF}}=244.5 \mathrm{~Hz}, \mathrm{C}-4^{\prime}$ ), 167.7 (C-2). Anal. Calcd for $\mathrm{C}_{14} \mathrm{H}_{14} \mathrm{FN}_{5}: \mathrm{C}$, 61.98; H, 5.20; N, 25.81. Found: C, 61.92; H, 5.29; N, 25.72.

4-Amino-2-propyl-7-(4-chlorophenyl)imidazo[1,2-a][1,3,5]triazine (5o). Light brown solid, yield 16\%, mp 294-295 ${ }^{\circ} \mathrm{C}$ (EtOH). ${ }^{1} \mathrm{H}$ NMR (300 MHz, DMSO- $\left.d_{6}\right): \delta 0.94\left(3 \mathrm{H}, \mathrm{t},{ }^{3} J=7.4 \mathrm{~Hz}, \mathrm{CH}_{3}\right), 1.76$ $\left(2 \mathrm{H}, \mathrm{m},{ }^{3} J=7.4 \mathrm{~Hz}, \mathrm{CH}_{2}\right), 2.60\left(2 \mathrm{H}, \mathrm{t},{ }^{3} J=7.4 \mathrm{~Hz}, \mathrm{CH}_{2}\right), 7.53(2 \mathrm{H}$, $\mathrm{d},{ }^{3} \mathrm{~J}=8.7 \mathrm{~Hz}, \mathrm{H}-3^{\prime}$ and $\left.\mathrm{H}-5^{\prime}\right), 7.85\left(2 \mathrm{H}, \mathrm{d},{ }^{3} \mathrm{~J}=8.9 \mathrm{~Hz}, \mathrm{H}-2^{\prime}\right.$ and $\mathrm{H}-$ $\left.6^{\prime}\right), 8.17(1 \mathrm{H}, \mathrm{s}, \mathrm{H}-6), 8.36\left(2 \mathrm{H}\right.$, br s, $\left.\mathrm{NH}_{2}\right) .{ }^{13} \mathrm{C}$ NMR $(75 \mathrm{MHz}$, DMSO- $\left.d_{6}\right): \delta 13.6\left(\mathrm{CH}_{3}\right), 20.4\left(\mathrm{CH}_{2}\right), 40.2\left(\mathrm{CH}_{2}\right), 102.1(\mathrm{C}-6), 126.9$ (C-2' and C-6'), $128.8\left(\mathrm{C}-3^{\prime}\right.$ and $\left.\mathrm{C}-5^{\prime}\right), 132.2\left(\mathrm{C}-1^{\prime}\right), 132.3\left(\mathrm{C}-4^{\prime}\right)$, 142.1 (C-7), $150.4 \& 150.8$ (C-4 and C-8a), 167.8 (C-2). Anal. Calcd for $\mathrm{C}_{14} \mathrm{H}_{14} \mathrm{ClN}_{5}$ : C, 58.44; H, 4.90; N, 24.34. Found: C, 58.37; H, $5.03 ; \mathrm{N}, 24.22$.

4-Amino-2-propyl-7-(4-methyphenyl)imidazo[1,2-a][1,3,5]triazine (5p). Light yellow solid, yield 50\%, mp 305-307 ${ }^{\circ} \mathrm{C}$ (EtOH). ${ }^{1} \mathrm{H}$ NMR (300 MHz, DMSO- $\left.d_{6}\right): \delta 0.94\left(3 \mathrm{H}, \mathrm{t},{ }^{3} J=7.4 \mathrm{~Hz}, \mathrm{CH}_{3}\right), 1.77$ $\left(2 \mathrm{H}, \mathrm{m},{ }^{3} J=7.4 \mathrm{~Hz}, \mathrm{CH}_{2}\right), 2.34\left(3 \mathrm{H}, \mathrm{s}, \mathrm{CH}_{3}\right), 2.60\left(2 \mathrm{H}, \mathrm{t},{ }^{3} J=\right.$ $\left.7.4 \mathrm{~Hz}, \mathrm{CH}_{2}\right), 7.27\left(2 \mathrm{H}, \mathrm{d},{ }^{3} J=7.3 \mathrm{~Hz}, \mathrm{H}-3^{\prime}\right.$ and $\left.\mathrm{H}-5^{\prime}\right), 7.75(2 \mathrm{H}, \mathrm{d}$, ${ }^{3} J=8.0 \mathrm{~Hz}, \mathrm{H}-2^{\prime}$ and $\left.\mathrm{H}-6^{\prime}\right), 8.10(1 \mathrm{H}, \mathrm{s}, \mathrm{H}-6), 8.30\left(2 \mathrm{H}\right.$, br s, $\mathrm{NH}_{2}$ ). ${ }^{13} \mathrm{C}$ NMR (75 MHz, DMSO- $\left.d_{6}\right): \delta 13.6\left(\mathrm{CH}_{3}\right), 20.4\left(\mathrm{CH}_{2}\right), 20.8$ $\left(\mathrm{CH}_{3}\right), 40.2\left(\mathrm{CH}_{2}\right), 101.2(\mathrm{C}-6), 125.2\left(\mathrm{C}^{\prime}\right)^{\prime}$ and $\left.\mathrm{C}-5^{\prime}\right), 129.3\left(\mathrm{C}-2^{\prime}\right.$ and C-6' $), 130.6\left(\mathrm{C}-1^{\prime}\right), 137.3\left(\mathrm{C}-4^{\prime}\right), 143.5(\mathrm{C}-7), 150.3 \& 150.7(\mathrm{C}-4$ and C-8a), 167.5 (C-2). Anal. Calcd for $\mathrm{C}_{15} \mathrm{H}_{17} \mathrm{~N}_{5}$ : C, 67.39; $\mathrm{H}$, 6.41; N, 26.20. Found: C, 67.32; H, 6.53; N, 26.06.

4-Amino-2-propyl-7-(4-methoxyphenyl)imidazo[1,2-a][1,3,5]triazine (5q). White solid, yield 52\%, mp 295-297 ${ }^{\circ} \mathrm{C}(\mathrm{MeOH}) .{ }^{1} \mathrm{H}$ NMR (300 MHz, DMSO- $\left.d_{6}\right): \delta 0.94\left(3 \mathrm{H}, \mathrm{t},{ }^{3} J=7.4 \mathrm{~Hz}, \mathrm{CH}_{3}\right), 1.76\left(2 \mathrm{H}, \mathrm{m},{ }^{3} J=\right.$ $\left.7.4 \mathrm{~Hz}, \mathrm{CH}_{2}\right), 2.59\left(2 \mathrm{H}, \mathrm{t},{ }^{3} \mathrm{~J}=7.4 \mathrm{~Hz}, \mathrm{CH}_{2}\right), 3.80\left(3 \mathrm{H}, \mathrm{s}, \mathrm{OCH}_{3}\right), 7.04$ $\left(2 \mathrm{H}, \mathrm{d},{ }^{3} J=8.9 \mathrm{~Hz}, \mathrm{H}-3^{\prime}\right.$ and $\left.\mathrm{H}-5^{\prime}\right), 7.78\left(2 \mathrm{H}, \mathrm{d},{ }^{3} J=8.9 \mathrm{~Hz}, \mathrm{H}-2^{\prime}\right.$ and $\left.\mathrm{H}^{\prime} 6^{\prime}\right), 8.03(1 \mathrm{H}, \mathrm{s}, \mathrm{H}-6), 8.30\left(2 \mathrm{H}, \mathrm{br} \mathrm{s}, \mathrm{NH}_{2}\right) .{ }^{13} \mathrm{C} \mathrm{NMR}(75 \mathrm{MHz}$, DMSO- $\left.d_{6}\right): \delta 13.6\left(\mathrm{CH}_{3}\right), 20.4\left(\mathrm{CH}_{2}\right), 40.2\left(\mathrm{CH}_{2}\right), 55.1\left(\mathrm{OCH}_{3}\right), 100.4$ (C-6), 114.2 (C-3' and C-5'), $125.9\left(\mathrm{C}^{\prime} \mathbf{1}^{\prime}\right), 126.6\left(\mathrm{C}-2^{\prime}\right.$ and C-6'), 143.4
(C-7), 150.3 \& 150.6 (C-4 and C-8a), 159.2 (C-4'), 167.3 (C-2). Anal. Calcd for $\mathrm{C}_{15} \mathrm{H}_{17} \mathrm{~N}_{5} \mathrm{O}$ : C, 63.59; H, 6.05; N, 24.72. Found: C, 63.52; $\mathrm{H}, 6.20$; N, 24.59.

4-Amino-2-propyl-7-(4-biphenyl)imidazo[1,2-a][1,3,5]triazine (5r). Light brown solid, yield 40\%, mp 337-338 ${ }^{\circ} \mathrm{C}$ (DMF). ${ }^{1} \mathrm{H}$ NMR (300 MHz, DMSO- $\left.d_{6}\right): \delta 0.95\left(3 \mathrm{H}, \mathrm{t},{ }^{3} J=7.4 \mathrm{~Hz}, \mathrm{CH}_{3}\right), 1.78\left(2 \mathrm{H}, \mathrm{m},{ }^{3} J=\right.$ $\left.7.4 \mathrm{~Hz}, \mathrm{CH}_{2}\right), 2.62\left(2 \mathrm{H}, \mathrm{t},{ }^{3} J=7.4 \mathrm{~Hz}, \mathrm{CH}_{2}\right), 7.38\left(1 \mathrm{H}, \mathrm{t},{ }^{3} J=7.3 \mathrm{~Hz}\right.$, $\left.\mathrm{H}-4^{\prime \prime}\right), 7.49\left(2 \mathrm{H}, \mathrm{t},{ }^{3} \mathrm{~J}=7.5 \mathrm{~Hz}, \mathrm{H}-3^{\prime \prime}\right.$ and $\left.\mathrm{H}-5^{\prime \prime}\right), 7.73\left(2 \mathrm{H}, \mathrm{d},{ }^{3} \mathrm{~J}=\right.$ $7.2 \mathrm{~Hz}, \mathrm{H}-2^{\prime \prime}$ and $\left.\mathrm{H}-6^{\prime \prime}\right), 7.79\left(2 \mathrm{H}, \mathrm{d},{ }^{3} J=8.4 \mathrm{~Hz}, \mathrm{H}-3^{\prime}\right.$ and $\left.\mathrm{H}-5^{\prime}\right), 7.95$ $\left(2 \mathrm{H}, \mathrm{d},{ }^{3} J=8.3 \mathrm{~Hz}, \mathrm{H}-2^{\prime}\right.$ and $\left.\mathrm{H}-6^{\prime}\right), 8.21(1 \mathrm{H}, \mathrm{s}, \mathrm{H}-6), 8.36(2 \mathrm{H}, \mathrm{br} \mathrm{s}$, $\left.\mathrm{NH}_{2}\right) \cdot{ }^{13} \mathrm{C}$ NMR (75 MHz, DMSO- $\left.d_{6}\right): \delta 13.7\left(\mathrm{CH}_{3}\right), 20.4\left(\mathrm{CH}_{2}\right), 40.2$ $\left(\mathrm{CH}_{2}\right), 101.9$ (C-6), 125.8 (C-3' and C-5'), $126.4\left(\mathrm{C}-3^{\prime \prime}\right.$ and $\left.\mathrm{C}-5^{\prime \prime}\right)$, $127.0\left(\mathrm{C}-2^{\prime}\right.$ and C-6'), $127.4\left(\mathrm{C}-4^{\prime \prime}\right), 128.9\left(\mathrm{C}-2^{\prime \prime}\right.$ and C-6"), $132.4(\mathrm{C}-$ $\left.1^{\prime}\right), 139.5 \& 139.6\left(\mathrm{C}-4^{\prime}\right.$ and $\left.\mathrm{C}-1^{\prime \prime}\right), 143.0(\mathrm{C}-7), 150.4 \& 150.8(\mathrm{C}-4$ and C-8a), 167.7 (C-2). Anal. Calcd for $\mathrm{C}_{20} \mathrm{H}_{19} \mathrm{~N}_{5}$ : C, 72.92; H, 5.81; N, 21.26. Found: C, 72.88; H, 5.94; N, 21.13.

4-Amino-2-butyl-7-phenylimidazo[1,2-a][1,3,5]triazine

(5s). Light yellow solid, yield 30\%, mp 267-269 ${ }^{\circ} \mathrm{C}(\mathrm{EtOH}) .{ }^{1} \mathrm{H}$ NMR (300 MHz, DMSO- $\left.d_{6}\right): \delta 0.92\left(3 \mathrm{H}, \mathrm{t},{ }^{3} J=7.3 \mathrm{~Hz}, \mathrm{CH}_{3}\right), 1.36(2 \mathrm{H}$, $\left.\mathrm{m},{ }^{3} J=7.4 \mathrm{~Hz}, \mathrm{CH}_{2}\right), 1.72\left(2 \mathrm{H}, \mathrm{m},{ }^{3} J=7.5 \mathrm{~Hz}, \mathrm{CH}_{2}\right), 2.62(2 \mathrm{H}, \mathrm{t}$, $\left.{ }^{3} J=7.5 \mathrm{~Hz}, \mathrm{CH}_{2}\right), 7.35\left(1 \mathrm{H}, \mathrm{t},{ }^{3} J=7.3 \mathrm{~Hz}, \mathrm{H}-4^{\prime}\right), 7.47\left(2 \mathrm{H}, \mathrm{t},{ }^{3} J=\right.$ $7.5 \mathrm{~Hz}, \mathrm{H}-3^{\prime}$ and $\left.\mathrm{H}-5^{\prime}\right), 7.85\left(2 \mathrm{H}, \mathrm{d},{ }^{3} J=7.0 \mathrm{~Hz}, \mathrm{H}-2^{\prime}\right.$ and $\left.\mathrm{H}-6^{\prime}\right)$, 8.15 (1H, s, H-6), 8.35 (2H, br s, $\mathrm{NH}_{2}$ ). ${ }^{13} \mathrm{C}$ NMR (75 MHz, DMSO$\left.d_{6}\right): \delta 13.7\left(\mathrm{CH}_{3}\right), 21.7\left(\mathrm{CH}_{2}\right), 29.3\left(\mathrm{CH}_{2}\right), 37.9\left(\mathrm{CH}_{2}\right), 101.7(\mathrm{C}-6)$, $125.2\left(\mathrm{C}-2^{\prime}\right.$ and C-6 $), 127.9\left(\mathrm{C}-4^{\prime}\right), 128.7\left(\mathrm{C}-3^{\prime}\right.$ and C-5'), $133.3(\mathrm{C}-$ $1^{\prime}$ ), 143.3 (C-7), 150.3 \& 150.8 (C-4 and C-8a), 167.8 (C-2). Anal. Calcd for $\mathrm{C}_{15} \mathrm{H}_{17} \mathrm{~N}_{5}$ : C, 67.39; H, 6.41; N, 26.20. Found: C, 67.32; $\mathrm{H}, 6.55 ; \mathrm{N}, 26.04$.

4-Amino-2-butyl-7-(4-fluorophenyl)imidazo[1,2-a][1,3,5]triazine (5t). White solid, yield 29\%, mp 296-298 ${ }^{\circ} \mathrm{C}$ (EtOH). ${ }^{1} \mathrm{H}$ NMR (300 MHz, DMSO- $\left.d_{6}\right): \delta 0.92\left(3 \mathrm{H}, \mathrm{t},{ }^{3} J=7.3 \mathrm{~Hz}, \mathrm{CH}_{3}\right), 1.36(2 \mathrm{H}$, $\left.\mathrm{m},{ }^{3} J=7.4 \mathrm{~Hz}, \mathrm{CH}_{2}\right), 1.72\left(2 \mathrm{H}, \mathrm{m},{ }^{3} J=7.5 \mathrm{~Hz}, \mathrm{CH}_{2}\right), 2.62(2 \mathrm{H}, \mathrm{t}$, $\left.{ }^{3} J=7.5 \mathrm{~Hz}, \mathrm{CH}_{2}\right), 7.31\left(2 \mathrm{H}, \mathrm{dd},{ }^{3} J_{\mathrm{HF}}=8.9 \mathrm{~Hz},{ }^{3} J_{\mathrm{HH}}=8.9 \mathrm{~Hz}, \mathrm{H}-3^{\prime}\right.$ and $\left.\mathrm{H}^{-} 5^{\prime}\right), 7.88\left(2 \mathrm{H}, \mathrm{dd},{ }^{4} J_{\mathrm{HF}}=5.6 \mathrm{~Hz},{ }^{3} J_{\mathrm{HH}}=8.9 \mathrm{~Hz}, \mathrm{H}-2^{\prime}\right.$ and $\mathrm{H}-$ $\left.6^{\prime}\right), 8.12(1 \mathrm{H}, \mathrm{s}, \mathrm{H}-6), 8.34\left(2 \mathrm{H}, \mathrm{br} \mathrm{s}, \mathrm{NH}_{2}\right) .{ }^{13} \mathrm{C} \mathrm{NMR}(75 \mathrm{MHz}$, DMSO- $\left.d_{6}\right): \delta 13.8\left(\mathrm{CH}_{3}\right), 21.7\left(\mathrm{CH}_{2}\right), 29.3\left(\mathrm{CH}_{2}\right), 37.9\left(\mathrm{CH}_{2}\right), 101.5$ (C-6), $115.7\left(\mathrm{~d},{ }^{2} J_{\mathrm{CF}}=21.7 \mathrm{~Hz}, \mathrm{C}-3^{\prime}\right.$ and C-5 $), 127.2\left(\mathrm{~d},{ }^{3} J_{\mathrm{CF}}=\right.$ $8.3 \mathrm{~Hz}, \mathrm{C}-2^{\prime}$ and C-6 $\left.{ }^{\prime}\right), 129.9\left(\mathrm{~d},{ }^{4} J_{\mathrm{CF}}=3.0 \mathrm{~Hz}, \mathrm{C}-1^{\prime}\right), 142.4(\mathrm{C}-7)$, $150.4 \& 150.8$ (C-4 and C-8a), $161.9\left(\mathrm{~d},{ }^{1} J_{\mathrm{CF}}=244.8 \mathrm{~Hz}, \mathrm{C}-4^{\prime}\right)$, 167.9 (C-2). Anal. Calcd for $\mathrm{C}_{15} \mathrm{H}_{16} \mathrm{FN}_{5}: \mathrm{C}, 63.14 ; \mathrm{H}, 5.65 ; \mathrm{N}$, 24.55. Found: C, 63.05; H, 5.77; N, 24.42.

4-Amino-2-butyl-7-(4-chlorophenyl)imidazo[1,2-a][1,3,5]triazine (5u). Light brown solid, yield 11\%, mp 287-288 ${ }^{\circ} \mathrm{C}$ (EtOH). ${ }^{1} \mathrm{H}$ NMR (300 MHz, DMSO- $\left.d_{6}\right): \delta 0.91\left(3 \mathrm{H}, \mathrm{t},{ }^{3} J=7.3 \mathrm{~Hz}, \mathrm{CH}_{3}\right), 1.35$ $\left(2 \mathrm{H}, \mathrm{m},{ }^{3} \mathrm{~J}=7.4 \mathrm{~Hz}, \mathrm{CH}_{2}\right), 1.72\left(2 \mathrm{H}, \mathrm{m},{ }^{3} J=7.5 \mathrm{~Hz}, \mathrm{CH}_{2}\right), 2.62$ $\left(2 \mathrm{H}, \mathrm{t},{ }^{3} J=7.5 \mathrm{~Hz}, \mathrm{CH}_{2}\right), 7.53\left(2 \mathrm{H}, \mathrm{d},{ }^{3} J=8.6 \mathrm{~Hz}, \mathrm{H}-3^{\prime}\right.$ and $\left.\mathrm{H}-5^{\prime}\right)$, $7.84\left(2 \mathrm{H}, \mathrm{d},{ }^{3} J=8.6 \mathrm{~Hz}, \mathrm{H}-2^{\prime}\right.$ and $\left.\mathrm{H}-6^{\prime}\right), 8.16(1 \mathrm{H}, \mathrm{s}, \mathrm{H}-6), 8.36$ $\left(2 \mathrm{H}\right.$, br s, $\left.\mathrm{NH}_{2}\right) \cdot{ }^{13} \mathrm{C} \mathrm{NMR}\left(75 \mathrm{MHz}, \mathrm{DMSO}-d_{6}\right): \delta 13.7\left(\mathrm{CH}_{3}\right), 21.7$ $\left(\mathrm{CH}_{2}\right), 29.3\left(\mathrm{CH}_{2}\right), 37.9\left(\mathrm{CH}_{2}\right), 102.1$ (C-6), 126.9 (C-2' and C-6'), $128.8\left(\mathrm{C}-3^{\prime}\right.$ and $\left.\mathrm{C}-5^{\prime}\right), 132.2 \& 132.33\left(\mathrm{C}-1^{\prime}\right.$ and $\left.\mathrm{C}-4^{\prime}\right), 142.1(\mathrm{C}-7)$, $150.4 \& 150.8$ (C-4 and C-8a), 168.1 (C-2). Anal. Calcd for $\mathrm{C}_{15} \mathrm{H}_{16} \mathrm{ClN}_{5}$ : C, 59.70; H, 5.34; N, 23.21. Found: C, 59.63; H, $5.41 ; \mathrm{N}, 23.15$.

4-Amino-2-butyl-7-(4-methylphenyl)imidazo[1,2-a][1,3,5]triazine (5v). Light yellow solid, yield 25\%, mp 287-289 ${ }^{\circ} \mathrm{C}(\mathrm{EtOH}) .{ }^{1} \mathrm{H}$ NMR (300 MHz, DMSO- $\left.d_{6}\right): \delta 0.92\left(3 \mathrm{H}, \mathrm{t},{ }^{3} J=7.3 \mathrm{~Hz}, \mathrm{CH}_{3}\right), 1.36$ $\left(2 \mathrm{H}, \mathrm{m},{ }^{3} \mathrm{~J}=7.4 \mathrm{~Hz}, \mathrm{CH}_{2}\right), 1.72\left(2 \mathrm{H}, \mathrm{m},{ }^{3} J=7.5 \mathrm{~Hz}, \mathrm{CH}_{2}\right), 2.34$ 
$\left(3 \mathrm{H}, \mathrm{s}, \mathrm{CH}_{3}\right), 2.62\left(2 \mathrm{H}, \mathrm{t},{ }^{3} J=7.5 \mathrm{~Hz}, \mathrm{CH}_{2}\right), 7.27\left(2 \mathrm{H}, \mathrm{d},{ }^{3} J=\right.$ $7.9 \mathrm{~Hz}, \mathrm{H}-3^{\prime}$ and $\left.\mathrm{H}-5^{\prime}\right), 7.74\left(2 \mathrm{H}, \mathrm{d},{ }^{3} J=8.1 \mathrm{~Hz}, \mathrm{H}-2^{\prime}\right.$ and $\left.\mathrm{H}-6^{\prime}\right)$, $8.09(1 \mathrm{H}, \mathrm{s}, \mathrm{H}-6), 8.30\left(2 \mathrm{H}\right.$, br s, $\left.\mathrm{NH}_{2}\right) .{ }^{13} \mathrm{C}$ NMR (75 MHz, DMSO$\left.d_{6}\right): \delta 13.7\left(\mathrm{CH}_{3}\right), 20.8\left(\mathrm{CH}_{3}\right), 21.7\left(\mathrm{CH}_{2}\right), 29.3\left(\mathrm{CH}_{2}\right), 37.9\left(\mathrm{CH}_{2}\right)$, 101.2 (C-6), 125.2 (C-2' and C-6'), 129.3 (C-3' and C-5'), 130.5 (C$\left.1^{\prime}\right), 137.3\left(\mathrm{C}-4^{\prime}\right), 143.5$ (C-7), 150.3 \& 150.7 (C-4 and C-8a), 167.7 (C-2). Anal. Calcd for $\mathrm{C}_{16} \mathrm{H}_{19} \mathrm{~N}_{5}$ : C, 68.30; H, 6.81; N, 24.89. Found: C, 68.24; H, 6.90; N, 24.81.

4-Amino-2-butyl-7-(4-methoxyphenyl)imidazo[1,2-a][1,3,5]triazine (5w). Light yellow solid, yield 25\%, mp 279-281 ${ }^{\circ} \mathrm{C}(\mathrm{MeOH}) .{ }^{1} \mathrm{H}$ NMR (300 MHz, DMSO- $\left.d_{6}\right): \delta 0.92\left(3 \mathrm{H}, \mathrm{t},{ }^{3} J=7.3 \mathrm{~Hz}, \mathrm{CH}_{3}\right), 1.36$ $\left(2 \mathrm{H}, \mathrm{m},{ }^{3} J=7.4 \mathrm{~Hz}, \mathrm{CH}_{2}\right), 1.72\left(2 \mathrm{H}, \mathrm{m},{ }^{3} J=7.5 \mathrm{~Hz}, \mathrm{CH}_{2}\right), 2.62(2 \mathrm{H}$, $\left.\mathrm{t},{ }^{3} J=7.5 \mathrm{~Hz}, \mathrm{CH}_{2}\right), 3.81\left(3 \mathrm{H}, \mathrm{s}, \mathrm{OCH}_{3}\right), 7.04\left(2 \mathrm{H}, \mathrm{d},{ }^{3} J=8.9 \mathrm{~Hz}, \mathrm{H}^{-}\right.$ $3^{\prime}$ and $\left.\mathrm{H}-5^{\prime}\right), 7.78\left(2 \mathrm{H}, \mathrm{d},{ }^{3} J=8.8 \mathrm{~Hz}, \mathrm{H}-2^{\prime}\right.$ and $\left.\mathrm{H}^{-} 6^{\prime}\right), 8.03\left(1 \mathrm{H}, \mathrm{s}, \mathrm{H}^{-}\right.$ 6), $8.30\left(2 \mathrm{H}\right.$, br s, $\left.\mathrm{NH}_{2}\right) .{ }^{13} \mathrm{C}$ NMR (75 MHz, DMSO- $\left.d_{6}\right): \delta 13.8$ $\left(\mathrm{CH}_{3}\right), 21.7\left(\mathrm{CH}_{2}\right), 29.3\left(\mathrm{CH}_{2}\right), 37.9\left(\mathrm{CH}_{2}\right), 55.1\left(\mathrm{OCH}_{3}\right), 100.4(\mathrm{C}-6)$, $114.2\left(\mathrm{C}-3^{\prime}\right.$ and $\left.\mathrm{C}-5^{\prime}\right), 125.9\left(\mathrm{C}-1^{\prime}\right), 126.6\left(\mathrm{C}-2^{\prime}\right.$ and C-6'), $143.4(\mathrm{C}-$ 7), 150.3 \& 150.6 (C-4 and C-8a), 159.2 (C-4'), 167.6 (C-2). Anal. Calcd for $\mathrm{C}_{16} \mathrm{H}_{19} \mathrm{~N}_{5} \mathrm{O}$ : C, 64.63; H, 6.44; N, 23.55. Found: C, 64.55; $\mathrm{H}, 6.54 ; \mathrm{N}, 23.43$.

4-Amino-2-butyl-7-(4-biphenyl)imidazo[1,2-a][1,3,5]triazine (5x). Light brown solid, yield 43\%, mp 327-329 ${ }^{\circ} \mathrm{C}$ (DMF). ${ }^{1} \mathrm{H}$ NMR (300 MHz, DMSO- $\left.d_{6}\right): \delta 0.92\left(3 \mathrm{H}, \mathrm{t},{ }^{3} J=7.3 \mathrm{~Hz}, \mathrm{CH}_{3}\right), 1.37\left(2 \mathrm{H}, \mathrm{m},{ }^{3} J=\right.$ $\left.7.4 \mathrm{~Hz}, \mathrm{CH}_{2}\right), 1.73\left(2 \mathrm{H}, \mathrm{m},{ }^{3} J=7.5 \mathrm{~Hz}, \mathrm{CH}_{2}\right), 2.63\left(2 \mathrm{H}, \mathrm{t},{ }^{3} J=7.5 \mathrm{~Hz}\right.$, $\left.\mathrm{CH}_{2}\right), 7.38\left(1 \mathrm{H}, \mathrm{t},{ }^{3} J=7.3 \mathrm{~Hz}, \mathrm{H}-4^{\prime \prime}\right), 7.49\left(2 \mathrm{H}, \mathrm{t},{ }^{3} J=7.5 \mathrm{~Hz}, \mathrm{H}-3^{\prime \prime}\right.$ and $\left.\mathrm{H}-5^{\prime \prime}\right), 7.73\left(2 \mathrm{H}, \mathrm{d},{ }^{3} J=7.5 \mathrm{~Hz}, \mathrm{H}-2^{\prime \prime}\right.$ and $\left.\mathrm{H}-6^{\prime \prime}\right), 7.79\left(2 \mathrm{H}, \mathrm{d},{ }^{3} J=\right.$ $8.4 \mathrm{~Hz}, \mathrm{H}-3^{\prime}$ and $\left.\mathrm{H}-5^{\prime}\right), 7.94\left(2 \mathrm{H}, \mathrm{d},{ }^{3} J=8.4 \mathrm{~Hz}, \mathrm{H}-2^{\prime}\right.$ and $\left.\mathrm{H}-6^{\prime}\right), 8.20$ (1H, s, H-6), 8.36 (2H, br s, $\mathrm{NH}_{2}$ ). ${ }^{13} \mathrm{C}$ NMR (75 MHz, DMSO- $d_{6}$ ): $\delta 13.7\left(\mathrm{CH}_{3}\right), 21.7\left(\mathrm{CH}_{2}\right), 29.3\left(\mathrm{CH}_{2}\right), 37.9\left(\mathrm{CH}_{2}\right), 101.8(\mathrm{C}-6), 125.8$ $\left(\mathrm{C}-3^{\prime}\right.$ and C-5'), 126.4 (C-3" and C-5" $\left.{ }^{\prime \prime}\right), 127.0\left(\mathrm{C}-2^{\prime}\right.$ and C-6' $), 127.4$ $\left(\mathrm{C}-4^{\prime \prime}\right), 128.9$ (C-2" and C-6"), $132.4\left(\mathrm{C}-1^{\prime}\right), 139.5$ (C-4' and C-1"), 143.0 (C-7), 150.4 \& 150.7 (C-4 and C-8a), 167.9 (C-2). Anal. Calcd for $\mathrm{C}_{21} \mathrm{H}_{21} \mathrm{~N}_{5}$ : C, 73.44; $\mathrm{H}, 6.16 ; \mathrm{N}, 20.39$. Found: $\mathrm{C}, 73.38 ; \mathrm{H}$, $6.26 ; \mathrm{N}, 20.31$.

Synthesis of 4-amino-2-methyl-7-phenylimidazo[1,2-a $][1,3,5]$ triazine (5a) using Monowave 400 (Anton Paar) microwave synthesizer. The mixture of 2-amino-4-phenyl- $1 H$-imidazole (1a, $159 \mathrm{mg}, 1 \mathrm{mmol})$, cyanamide (105 $\mathrm{mg}, 2.5 \mathrm{mmol}$ ) and triethyl orthoacetate $(0.46 \mathrm{~mL}, 2.5 \mathrm{mmol})$ in ethyl acetate $(2 \mathrm{~mL})$ were irradiated in a $10 \mathrm{~mL}$ seamless pressure vial using a microwave system operating at maximal microwave power up to $850 \mathrm{~W}$ (Monowave 400, Anton Paar) at $160{ }^{\circ} \mathrm{C}$ for $35 \mathrm{~min}$. After cooling, the precipitate was filtered and washed with ethyl acetate to give $5 \mathbf{a}$ in the $56 \%$ yield.

Synthesis of 4-amino-2-methyl-7-phenylimidazo[1,2-a][1,3,5] triazine (5a) via heating in a sealed vessel. The mixture of 2amino-4-phenyl-1 $H$-imidazole (1a, $159 \mathrm{mg}, 1 \mathrm{mmol}$ ), cyanamide (105 mg, $2.5 \mathrm{mmol})$ and triethyl orthoacetate $(0.46 \mathrm{~mL}, 2.5$ $\mathrm{mmol})$ in ethyl acetate $(2 \mathrm{~mL})$ were heated in a $10 \mathrm{~mL}$ seamless pressure vial in an enclosed system (Monowave 50, Anton Paar) at $160{ }^{\circ} \mathrm{C}$ for $35 \mathrm{~min}$. After cooling, the precipitate was filtered to obtain a compound, which was identical to 5a prepared under microwave irradiation. Yield 54\%.

Step-wise approach for the synthesis of 4-amino-2-methyl-7phenylimidazo $[1,2-a][1,3,5]$ triazine (5a)

$N^{\prime}, N^{\prime}$-Morpholino- $N-[3(5)$-phenylimidazolo-5(3)yl acetamidine (6). A mixture of 2-amino- $1 H$-imidazole 1a (159 mg, $1 \mathrm{mmol})$ with triethyl orthoacetate $(0.46 \mathrm{~mL}, 2.5 \mathrm{mmol})$ and morpholine $(0.22$ $\mathrm{mL}, 2.5 \mathrm{mmol}$ ) in ethyl acetate $(2 \mathrm{~mL})$ were irradiated in a $10 \mathrm{~mL}$ seamless pressure vial using the Discover SP (CEM) microwave system operating at maximal microwave power up to $150 \mathrm{~W}$ at $160{ }^{\circ} \mathrm{C}$ for $35 \mathrm{~min}$. After cooling, the precipitate was filtered and recrystallised from $\mathrm{EtOH}$ to give pure formamidine 6. Light yellow solid, yield 34\%, mp 217-219 ${ }^{\circ} \mathrm{C}$ (EtOH). ${ }^{1} \mathrm{H}$ NMR $(300 \mathrm{MHz}$, DMSO- $\left.d_{6}\right): \delta 2.28\left(3 \mathrm{H}, \mathrm{s}, \mathrm{CH}_{3}\right), 3.55-3.56\left(4 \mathrm{H}, \mathrm{m},\left(\mathrm{CH}_{2}\right)_{2}\right), 3.62-3.65$ $\left(4 \mathrm{H}, \mathrm{m},\left(\mathrm{CH}_{2}\right)_{2}\right), 7.10\left(1 \mathrm{H}, \mathrm{t},{ }^{3} J=7.3 \mathrm{~Hz}, \mathrm{H}-4^{\prime}\right), 7.22(1 \mathrm{H}, \mathrm{s}, \mathrm{CH}), 7.29$ $\left(2 \mathrm{H}, \mathrm{t},{ }^{3} \mathrm{~J}=7.7 \mathrm{~Hz}, \mathrm{H}-3^{\prime}\right.$ and $\left.\mathrm{H}-5^{\prime}\right), 7.66\left(2 \mathrm{H}, \mathrm{d},{ }^{3} \mathrm{~J}=6.9 \mathrm{~Hz}, \mathrm{H}-2^{\prime}\right.$ and $\left.\mathrm{H}-6^{\prime}\right), 11.08$ \& $11.44\left(1 \mathrm{H}\right.$, br s, NH). Anal. Calcd for $\mathrm{C}_{15} \mathrm{H}_{18} \mathrm{~N}_{4} \mathrm{O}: \mathrm{C}$, 66.64; H, 6.71; N, 20.73. Found: C, 66.46; H, 6.94; N, 20.55.

4-Amino-2-methyl-7-phenylimidazo[1,2-a][1,3,5]triazine (5a). A mixture of 6 (270 mg, $1 \mathrm{mmol})$ with cyanamide (105 mg, 2.5 $\mathrm{mmol}$ ) in ethyl acetate $(2 \mathrm{~mL})$ were irradiated in a $10 \mathrm{~mL}$ seamless pressure vial using the Discover SP (CEM) microwave system operating at maximal microwave power up to $150 \mathrm{~W}$ at $160{ }^{\circ} \mathrm{C}$ for $35 \mathrm{~min}$. After cooling, the precipitate was filtered to obtain a compound, which was identical to 5a prepared using the multicomponent reaction. Yield $43 \%$.

X-Ray crystallographic analysis. Intensity data for $\mathbf{5 g}$ and $\mathbf{5 p}$ were measured at $100 \mathrm{~K}$ on an Rigaku/Oxford Diffraction XtaLAB Synergy diffractometer (Dualflex, AtlasS2) fitted with $\mathrm{CuK} \alpha$ radiation $\left(\lambda=1.54178 \AA\right.$ ) so that $\theta_{\max }=67.1^{\circ}$. Data reduction and Gaussian absorption corrections were by standard methods. ${ }^{22}$ The structures were solved by direct-methods ${ }^{23}$ and refined on $F^{2}$ (ref. 24) with anisotropic displacement parameters, $\mathrm{C}$-bound $\mathrm{H}$ atoms in the riding model approximation and $\mathrm{N}$-bound $\mathrm{H}$ atoms refined with $\mathrm{N}-\mathrm{H}=0.88 \pm 0.01 \AA$. A weighting scheme of the form $w=1 /\left[\sigma^{2}\left(F_{\mathrm{o}}{ }^{2}\right)+(a P)^{2}+b P\right]$ where $P=\left(F_{\mathrm{o}}{ }^{2}+2 F_{\mathrm{c}}{ }^{2}\right) / 3$ was introduced in each case. The molecular structure diagrams showing $70 \%$ probability displacement ellipsoids were generated by ORTEP for Windows ${ }^{25}$ and the packing diagrams with DIAMOND. ${ }^{26}$ Additional data analysis was made with PLATON. ${ }^{27}$

Crystal data for 4-amino-2-ethyl-7-phenylimidazo[1,2-a][1,3,5] triazine (5g). $M=239.28$, orthorhombic, Pccn, $a=16.6173(2)$, $b=11.17070(10), c=12.4074(2) \AA, V=2303.15(5) \AA^{3}, Z=8, D_{\mathrm{x}}=$ $1.380 \mathrm{~g} \mathrm{~cm}^{-3}, F(000)=1008, \mu=0.709 \mathrm{~mm}^{-1}$, no. reflns meas. $=14762$, no. unique reflns $=2060\left(R_{\text {int }}=0.026\right)$, no. reflns with $I \geq 2 \sigma(I)=1935$, no. parameters $=170, R($ obs. data $)=0.032$, $w R 2($ all data $)=0.085$. CCDC deposition number: 1840247 .

Crystal data for 4-amino-2-propyl-7-(4-methyphenyl)imidazo [1,2-a][1,3,5]triazine (5p). $M=267.33$, triclinic, $P \overline{1}, a=$ 10.7534(2), $b=11.8995(2), c=12.1124(3) \AA, \alpha=71.918(2), \beta=$ 68.962(2), $\gamma=78.712(2)^{\circ}, V=1368.93(5) \AA^{3}, Z=4, D_{\mathrm{x}}=$ $1.297 \mathrm{~g} \mathrm{~cm}^{-3}, F(000)=568, \mu=0.650 \mathrm{~mm}^{-1}$, no. reflns meas. $=$ 32816 , no. unique reflns $=4893\left(R_{\text {int }}=0.029\right)$, no. reflns with $I$ $\geq 2 \sigma(I)=4530$, no. parameters $=377, R$ (obs. data $)=0.034$, $w R 2($ all data $)=0.092$. CCDC deposition number: 1840248 .

\section{Conflicts of interest}

There are no conflicts to declare. 


\section{Acknowledgements}

This work is supported by the Ministry of Higher Education, Malaysia under Fundamental Research Grant Scheme (FRGS). We would like to thank Anton Paar Malaysia Sdn. Bhd. for their technical support. The Research Centre for Crystalline Materials (Sunway University) is thanked for the X-ray intensity data.

\section{Notes and references}

1 F. P. L. Lim, N. R. Halcovitch, E. R. T. Tiekink and A. V. Dolzhenko, Tetrahedron, 2018, 74, 1868-1879.

2 F. P. L. Lim and A. V. Dolzhenko, Eur. J. Med. Chem., 2014, 85, 371-390.

3 P. Dao, N. Smith, C. Tomkiewicz-Raulet, E. Yen-Pon, M. Camacho-Artacho, D. Lietha, J.-P. Herbeuval, X. Coumoul, C. Garbay and H. Chen, J. Med. Chem., 2015, 58, 237-251.

4 X. Jiao, D. J. Kopecky, J. Liu, J. Liu, J. C. Jaen, M. G. Cardozo, R. Sharma, N. Walker, H. Wesche, S. Li, E. Farrelly, S.-H. Xiao, Z. Wang and F. Kayser, Bioorg. Med. Chem. Lett., 2012, 22, 6212-6217.

5 J. Feng, S. L. Gwaltney, J. A. Stafford, M. B. Wallace and Z. Zhang, US Pat., 20060135767, 2006.

6 H. Haning, U. Niewoehner, T. Schenke, T. Lampe, A. Hillisch and E. Bischoff, Bioorg. Med. Chem. Lett., 2005, 15, 39003907.

7 (a) M. Rzadkowska, E. Szacon, D. Matosiuk, E. Kedzierska and S. Fidecka, Acta Pol. Pharm., 2012, 69, 1270-1275; (b) D. Matosiuk, S. Fidecka, L. Antkiewicz-Michaluk, J. Lipkowski, I. Dybala and A. E. Koziol, Eur. J. Med. Chem., 2002, 37, 761-772.

8 E. Novellino, E. Abignente, B. Cosimelli, G. Greco, M. Iadanza, S. Laneri, A. Lavecchia, M. G. Rimoli, F. Da Settimo, G. Primofiore, D. Tuscano, L. Trincavelli and C. Martini, J. Med. Chem., 2002, 45, 5030-5036.

9 (a) F. Saczewski, M. Maruszak and P. J. Bednarski, Arch. Pharm., 2008, 341, 121-125; (b) I. Lakomska, B. Golankiewicz, J. Wietrzyk, M. Pelczynska, A. Nasulewicz, A. Opolski, J. Sitkowski, L. Kozerski and E. Szlyk, Inorg. Chim. Acta, 2005, 358, 1911-1917.

10 (a) D. Dukhan, F. Leroy, J. Peyronnet, E. Bosc, D. Chaves, M. Durka, R. Storer, P. La Colla, F. Seela and G. Gosselin, Nucleosides, Nucleotides Nucleic Acids, 2005, 24, 671-674; (b) J. O. Ojwang, S. Ali, D. F. Smee, J. D. Morrey, C. D. Shimasaki and R. W. Sidwell, Antiviral Res., 2005, 68, 49-55.

11 P. Cui, T. L. Macdonald, M. Chen and J. L. Nadler, Bioorg. Med. Chem. Lett., 2006, 16, 3401-3405.

12 J. Kobe, B. Stanovnik and M. Tisler, Chem. Commun., 1968, 1456.

13 (a) Z. Wang, H. K. Huynh, B. Han, R. Krishnamurthy and A. Eschenmoser, Org. Lett., 2003, 5, 2067-2070; (b) P. Rao and S. A. Benner, J. Org. Chem., 2001, 66, 5012-5015; (c) E. J. Prisbe, J. P. H. Verheyden and J. G. Moffatt, J. Org. Chem., 1978, 43, 4784-4794; (d) J. J. Voegel, M. M. Altorfer and S. A. Benner, Helv. Chim. Acta, 1993, 76, 2061-2069.
14 (a) P. Dao, C. Garbay and H. Chen, Tetrahedron, 2013, 69, 3867-3871; (b) T. Hanami, H. Oda, A. Nakamura, H. Urata, M. Itoh and Y. Hayashizaki, Tetrahedron Lett., 2007, 48, 3801-3803; (c) J. J. Li, C. Song, D.-M. Cui and C. Zhang, Org. Biomol. Chem., 2017, 15, 5564-5570; (d) L. B. Krasnova, J. E. Hein and V. V. Fokin, J. Org. Chem., 2010, 75, 8662-8665; (e) B. Han, B. Jaun, R. Krishnamurthy and A. Eschenmoser, Org. Lett., 2004, 6, 3691-3694; $(f)$ Z. Wang, H. K. Huynh, B. Han, R. Krishnamurthy and A. Eschenmoser, Org. Lett., 2003, 5, 2067-2070.

15 (a) J. F. Allochio Filho, B. C. Lemos, A. S. de Souza, S. Pinheiro and S. J. Greco, Tetrahedron, 2017, 73, 69777004; (b) S. Maddila, S. B. Jonnalagadda, K. K. Gangu and S. N. Maddila, Curr. Org. Synth., 2017, 14, 634-653; (c) J.-P. Wan, L. Gan and Y. Liu, Org. Biomol. Chem., 2017, 15, 9031-9043; (d) I. A. Ibarra, A. Islas-Jacome and E. Gonzalez-Zamora, Org. Biomol. Chem., 2018, 16, 14021418; (e) P. Patil, B. Mishra, G. Sheombarsing, K. Kurpiewska, J. Kalinowska-Tluscik and A. Doemling, ACS Comb. Sci., 2018, 20, 70-74; (f) E. A. Muravyova, S. M. Desenko, R. V. Rudenko, S. V. Shishkina, O. V. Shishkin, Y. V. Sen'ko, E. V. Vashchenko and V. A. Chebanov, Tetrahedron, 2011, 67, 9389-9400; $(g)$ P. V. Santhini, R. A. Krishnan, S. A. Babu, B. S. Simethy, G. Das, V. K. Praveen, S. Varughese and J. John, J. Org. Chem., 2017, 82, 10537-10548.

16 (a) R. Pathoor and D. Bahulayan, New J. Chem., 2018, 42, 6810-6816; (b) E. Ezzatzadeh and Z. Hossaini, Nat. Prod. Res., 2018, DOI: 10.1080/14786419.2018.1428598; (c) P. Sathiyachandran, P. Manogaran, V. N. Nesterov, V. V. Padma and K. J. Rajendra Prasad, Eur. J. Med. Chem., 2018, 150, 851-863; (d) P. R. Mali, P. K. Shirsat, N. Khomane, L. Nayak, J. B. Nanubolu and H. M. Meshram, ACS Comb. Sci., 2017, 19, 633-639.

17 (a) A. J. Ansari, S. Sharma, R. S. Pathare, K. Gopal, D. M. Sawant and R. T. Pardasani, ChemistrySelect, 2016, 1, 1016-1021; (b) A. S. Medvedeva, M. M. Demina, T. V. Kon'kova, T. L. H. Nguyen, A. V. Afonin and I. A. Ushakov, Tetrahedron, 2017, 73, 3979-3985; (c) N. M. Drosos, C. Kakoulidou, M. Raftopoulou, J. Stephanidou-Stephanatou, C. A. Tsoleridis and A. G. Hatzidimitriou, Tetrahedron, 2017, 73, 1-7; (d) F. B. Souza, D. C. Pimenta, H. A. Stefani and A. Helio, Tetrahedron Lett., 2016, 57, 1592-1596; (e) P. Vincetti, A. Brianza, N. Scalacci, G. Costantino, D. Castagnolo and M. Radi, Tetrahedron Lett., 2016, 57, 1464-1467; (f) M. Veeranarayana Reddy, B. Siva Kumar, K. T. Lim, B. G. Cho and Y. T. Jeong, Tetrahedron Lett., 2016, 57, 476478; $(g)$ A. Kumbhar, S. Jadhav, R. Shejwal, G. Rashinkar and R. Salunkhe, RSC Adv., 2016, 6, 19612-19619; (h) R. Mishra and L. H. Choudhury, RSC Adv., 2016, 6, 2446424469; (i) B. Jiang, T.-S. Zhang, R. Fu, W.-J. Hao, S.-L. Wang and S.-J. Tu, Tetrahedron, 2016, 72, 5652-5658; (j) M. Kumar, T. Kaur, V. K. Gupta and A. Sharma, RSC Adv., 2015, 5, 17087-17095; (k) P. Wadhwa, T. Kaur and A. Sharma, $R S C$ Adv., 2015, 5, 44353-44360. 
18 (a) F. P. L. Lim, G. Luna and A. V. Dolzhenko, Tetrahedron Lett., 2014, 55, 5159-5163; (b) F. P. L. Lim, G. Luna and A. V. Dolzhenko, Tetrahedron Lett., 2015, 56, 7016-7019; (c) F. P. L. Lim, G. Luna and A. V. Dolzhenko, Tetrahedron Lett., 2015, 56, 521-524; (d) A. V. Dolzhenko, S. A. Kalinina and D. V. Kalinin, RSC Adv., 2013, 3, 15850-15855; (e) S. A. Kalinina, D. V. Kalinin and A. V. Dolzhenko, Tetrahedron Lett., 2013, 54, 5537-5540; (f) F. P. L. Lim, S. T. Low, E. L. K. Ho, N. R. Halcovitch, E. R. T. Tiekink and A. V. Dolzhenko, RSC Adv., 2017, 7, 51062-51068.

19 (a) D. S. Ermolatev, E. P. Svidritsky, E. V. Babaev and E. Van der Eycken, Tetrahedron Lett., 2009, 50, 5218-5220; (b) D. S. Ermolat'ev and E. V. Van der Eycken, J. Org. Chem., 2008, 73, 6691-6697; (c) D. S. Ermolat'ev, B. Savaliya, A. Shah and E. Van der Eycken, Mol. Diversity, 2011, 15, 491-496.
20 D. V. Kalinin, S. A. Kalinina and A. V. Dolzhenko, Heterocycles, 2013, 87, 147-154.

21 Y. S. Agasimundin, F. T. Oakes and N. J. Leonard, J. Org. Chem., 1984, 50, 2474-2480.

22 Rigaku Oxford Diffraction, CrysAlis PRO, Yarnton, Oxfordshire, England, 2017.

23 G. M. Sheldrick, Acta Crystallogr., Sect. A: Found. Crystallogr., 2008, 64, 112-122.

24 L. J. Farrugia, J. Appl. Crystallogr., 2012, 45, 849-854.

25 G. M. Sheldrick, Acta Crystallogr., Sect. C: Struct. Chem., 2015, 71, 3-8.

26 K. Brandenburg, DIAMOND, Crystal Impact GbR, Bonn, Germany, 2006.

27 A. L. Spek, Acta Crystallogr., Sect. D: Biol. Crystallogr., 2009, 65, 148-155. 